Division of Geological \& Geophysical Surveys

RAW-DATA FILE 2000-4

\title{
MAJOR OXIDE, MINOR OXIDE, TRACE ELEMENT, RARE-EARTH ELEMENT, AND GEOCHEMICAL DATA FROM ROCKS COLLECTED IN EAGLE AND TANACROSS QUADRANGLES, ALASKA IN 2000
}

\author{
by \\ M.B. Werdon, D.J. Szumigala, R.J. Newberry, J.C. Grady, and W.C. Munly
}

October 2000

THIS REPORT HAS NOT BEEN REVIEWED FOR

TECHNICAL CONTENT OR FOR CONFORMITY TO THE

EDITORIAL STANDARDS OF DGGS

Released by

STATE OF ALASKA

DEPARTMENT OF NATURAL RESOURCES

Division of Geological \& Geophysical Surveys

794 University Avenue, Suite 200

Fairbanks, Alaska 99709-3645 


\section{CONTENTS}

PAGE

INTRODUCTION

ANALYTICAL METHODS

1

TABLES

TABLE 1. LOCATION AND DESCRIPTION OF ROCK SAMPLES COLLECTED IN THE EAGLE QUADRANGLE FOR TRACE ELEMENT GEOCHEMICAL ANALYSES.

TABLE 2. CONCENTRATION OF TRACE ELEMENTS IN ROCK SAMPLES COLLECTED IN THE EAGLE QUADRANGLE

TABLE 3. LOCATION AND DESCRIPTION OF ROCK SAMPLES COLLECTED IN THE EAGLE AND TANACROSS QUADRANGLES FOR MAJOR OXIDE, MINOR OXIDE, TRACE, AND RAREEARTH ELEMENT ANALYSES.

TABLE 4. CONCENTRATION OF MAJOR OXIDES, MINOR OXIDES, AND TRACE ELEMENTS IN ROCK SAMPLES COLLECTED IN THE EAGLE AND TANACROSS QUADRANGLES.

TABLE S. CONCENTRATION OF RARE-EARTH ELEMENTS IN ROCK SAMPLES COLLECTED IN THE EAGLE QUADRANGLE

TABLE 6. DETECTION LIMITS FOR TRACE-ELEMENT GEOCHEMICAL ANALYSES

TABLE 7. DETECTION LIMITS FOR MAJOR-OXIDE, MINOR-OXIDE, TRACE, AND RARE-EARTH ELEMENT ANALYSES

\section{SHEETS}

SHEET 1. LOCATION MAP OF ROCK SAMPLES ANALYZED FOR TRACE ELEMENT GEOCHEMISTRY, EAGLEA-1 AND A-2 QUADRANGLES, ALASKA IN 2000, 1:63,360 SCALE, I SHEET (IN POCKET)

SHEET 2. LOCATION MAP OF ROCK SAMPLES ANALYZED FOR MAJOR-OXIDES, MINOR OXIDES, TRACE ELEMENTS, AND (OR) RARE EARTH ELEMENTS, EAGLE A-1 AND A-2 QUADRANGLES, ALASKA IN 2000, 1:63,360 SCALE, I SHEET (IN POCKET)

SHEET 3. LOCATION MAP OF ROCK SAMPLES ANALYZED FOR TRACE ELEMENT GEOCHEMISTRY, MAJOR-OXIDES, MINOR OXIDES, TRACE ELEMENTS, AND (OR) RARE EARTH ELEMENTS, EAGLE B-3 QUADRANGLE, ALASKA IN 2000, 1:63,360 SCALE, 1 SHEET (IN POCKET)

Note: This report (including all analytical data, tables, and map sheets) is available in digital format from the DGGS web site (http://wwwdggs.dnr.state.ak.us) at no charge. The digital data are available as PDF files and Excel spreadsheets. 


\title{
MAJOR OXIDE, MINOR OXIDE, TRACE ELEMENT, RARE-EARTH ELEMENT, AND GEOCHEMICAL DATA FROM ROCKS COLLECTED IN THE EAGLE AND TANACROSS QUADRANGLES, ALASKA IN 2000
}

\author{
by
}

\author{
M.B. WERDON, D.J. SZUMIGALA, R.J. NEWBERRY, J.C. GRADY, AND W.C. MUNLY
}

\section{INTRODUCTION}

Mineral resource personnel from the Alaska Division of Geological \& Geophysical Surveys carried out a geological field survey, including mapping and sampling of Eagle and Tanacross quadrangles, Alaska from June 2, 2000 to July 12,2000 . The fieldwork provides basic information critical to building an understanding of Alaska's geology and is part of an integrated program of airbome geophysical surveys followed by geological mapping programs. During 2000, 157 rock samples were collected for geochemical trace-element analysis, and 96 samples were collected for whole rock (major and minor oxides, and petrogenetically important trace and (or) rare earth element data) analysis. The locations of these samples are shown on Sheets 1-3. Location data (in UTM coordinates with a Clark 1866, NAD27, UTM zone 7 projection), descriptions, and analytical results for each sample are tabulated in Tables 1-5.

\section{ANALYTICAL METHODS}

All 2000 trace-element geochemical analyses were performed by Chemex Labs, Inc. Rock samples were cnushed so that at least 70 percent of the material passed through a $-10(2 \mathrm{~mm})$ mesh screen. A 200-gram, representative split of the sample was then taken using a riffle splitter. The 200 -gram sample was then pulverized in a chrome steel ring mill so that 95 percent of the sample passed through a -150 (106 micron) mesh screen. DGGS also submitted geochemical standards at an approximate rate of one pulp per fifteen unknowns, and crushed granite and basalt standards were submined at an approximate rate of one whole rock standard per fifteen unknowns.

Gold was analyzed on a 30 gram representative sample split using Fire Assay (FA) and Atomic Absorption Spectroscopy (AAS) metbods. Most of the trace elements were analyzed by Inductively Coupled Plasma - Atomic Emission Spectroscopy (ICP-AES) methods after nitric aqua regia digestion. Platinum and palladium were analyzed by the Fire Assay - Inductively Coupled Plasma (FA-ICP) method. Analytical detection limits are tabulated in Table 6.

All whole rock analyses were performed by Chemex Labs, Inc. Major and minor element oxides were determined by XRF methods following a metaborate fusion. Trace elements ( $\mathrm{Ba}, \mathrm{Nb}, \mathrm{Rb}, \mathrm{Sr}, \mathrm{Y}$, and $\mathrm{Zr}$ ) were also analyzed using XRF methods on a pressed pellet. Rare Earth elements were analyzed using Inductively Coupled Plasma Mass spectroscopy after fusing with lithium metaborate. Analytical detection limits are tabulated in Table 7. 
Table 1. Location and description of rock samples collected in the Eagle quadrangle for trace element geochem ical analyses.

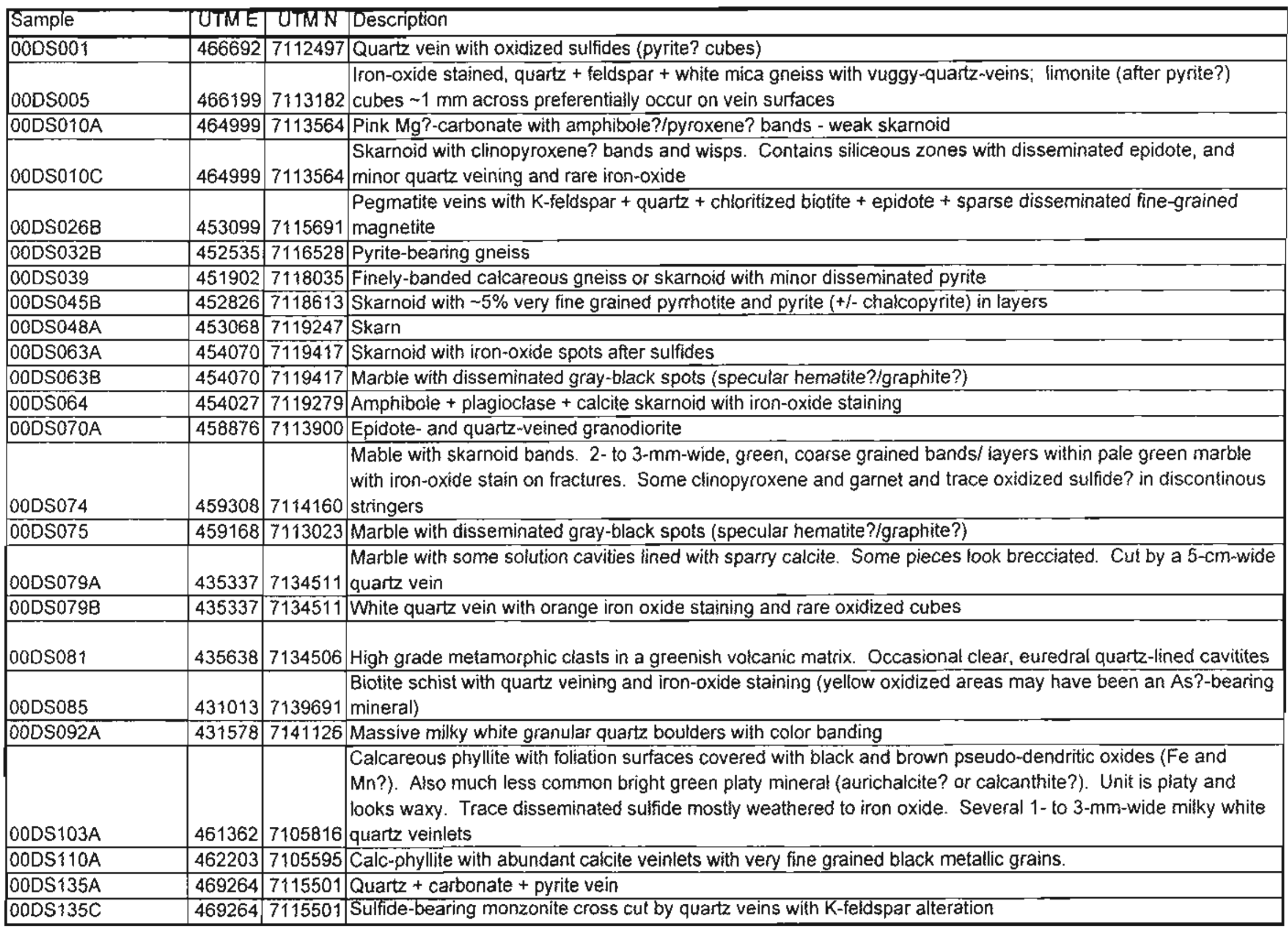


Table 1. Location and description of rock samples collected in the Eagle quadrangle for trace element geochemicaf analyses.

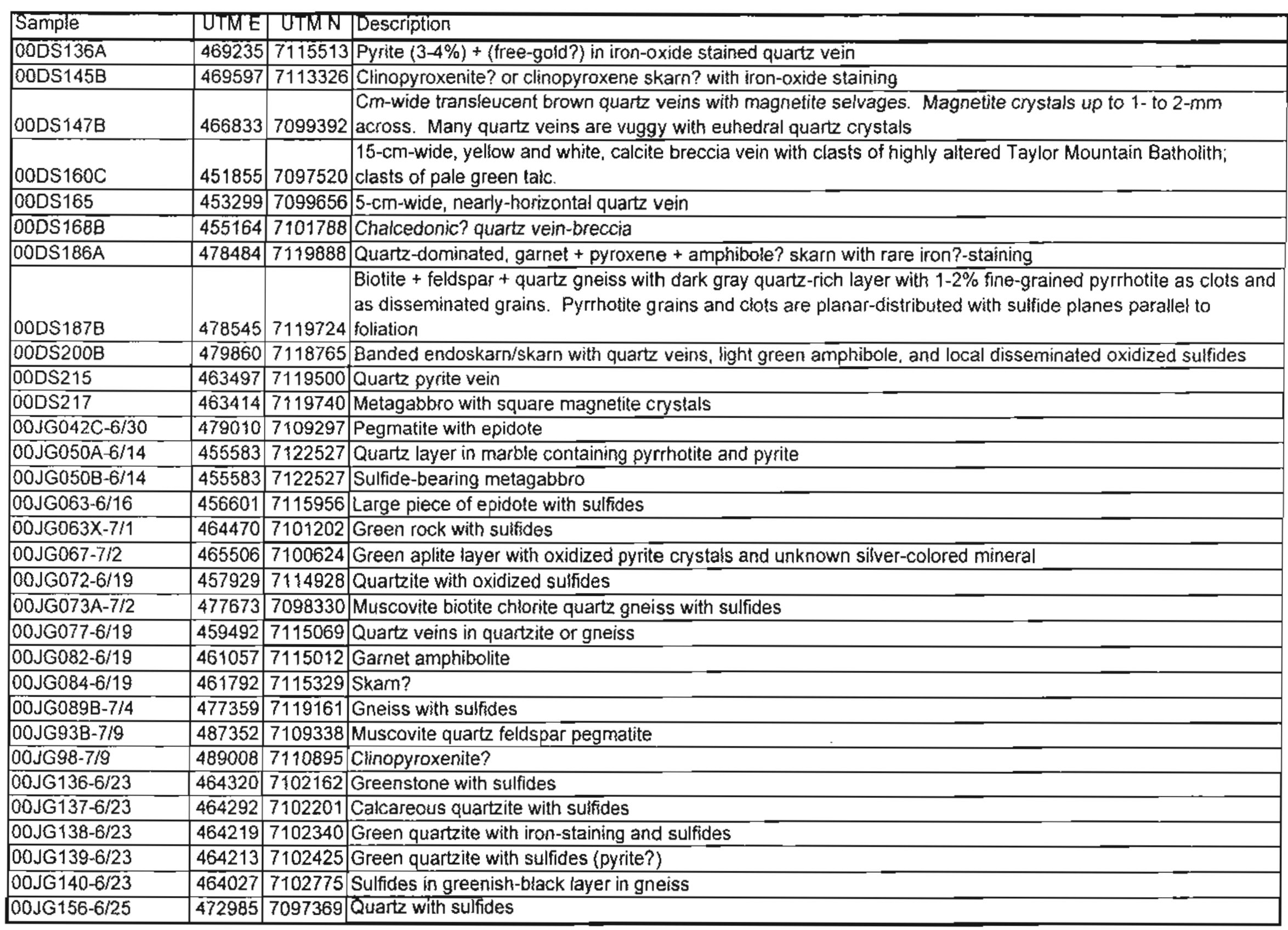


Table I. Location and description of rock samples collected in the Eagle quadrangle for trace element geochemical analyses.

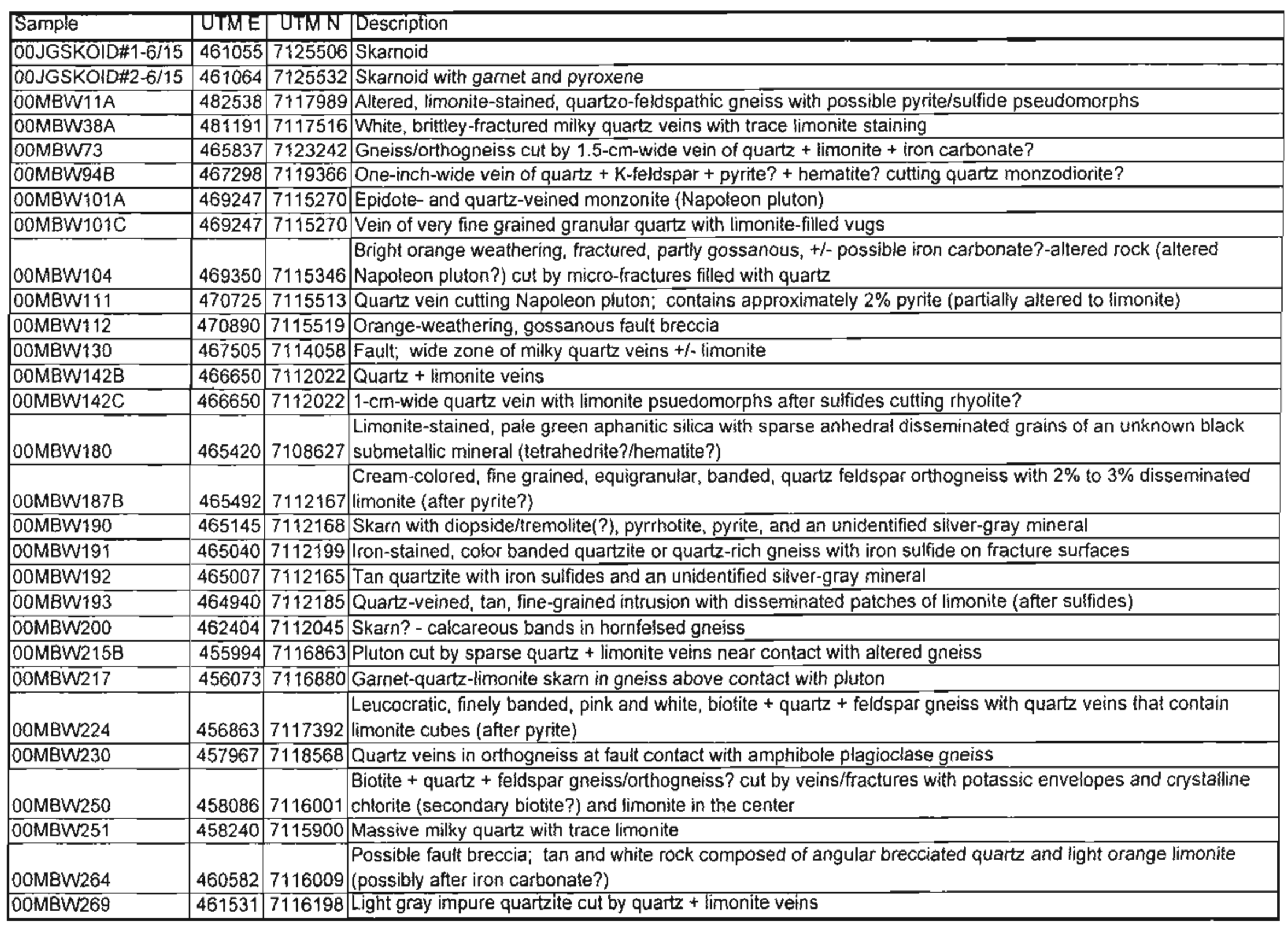


Table 1 . Location and description of rock samples collected in the Eagle quadrangle for Irace elenent geochemical analyses.

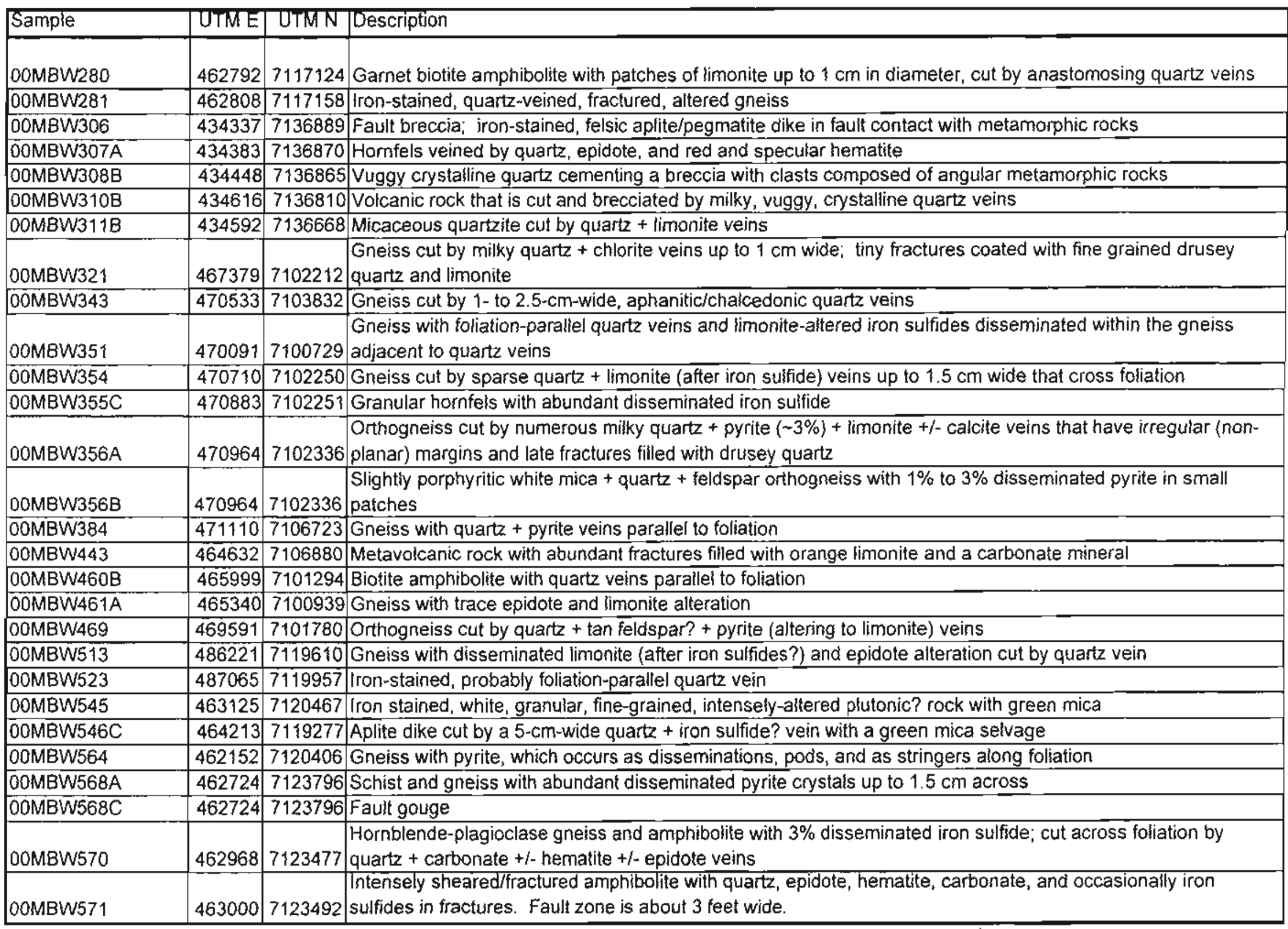


Table 1. Location and description of rock samples collected in the Eagle quadrangle for trace element geochemical analyses.

\begin{tabular}{|c|c|c|c|}
\hline Sample & UTME & UTMT & Description \\
\hline 00RN62 & 477451 & 7120611 & Quartz $+/$ - pyrite veinlets in gneiss \\
\hline OORN96C & 482502 & 7118013 & Orange-stained pyritic felsic schist - felsic metavolcanic? rock \\
\hline OORN102 & 484001 & $\overline{71+3448}$ & Brecciated, iron-stained quartzite \\
\hline OORN147B & 465555 & 7119920 & Skarn \\
\hline 0ORN $147 \mathrm{C}$ & $4655 \overline{55}$ & 7119920 & Mineralized quartz diorite \\
\hline DORN147D & 465555 & 7119920 & Mineralized granite pegmatite + aplite \\
\hline OORN175A & 452318 & 7121809 & 1-cm-thick pyroxene-actinolite skarn veins in marble \\
\hline OORN192A & 465488 & 7120009 & Pyroxene?-epidote? skam \\
\hline OORN192B & $4654 \overline{8} 8$ & 7120009 & Epidote-calcite and epidote-actinolite skarn \\
\hline OORN244A & $4540 \overline{05}$ & 7117870 & Skarn/skamoid \\
\hline OORN252A & 453524 & 7119335 & $\begin{array}{l}\text { Iron-stained skarn; 5-cm-wide, coarse-grained clinopyroxenite at marble front, adjacent to a coarse grained } \\
\text { amphibolite with up to } 5 \% \text { disseminated pyrite }\end{array}$ \\
\hline 00RN325 & 460454 & 7118283 & $\begin{array}{l}\text { Iron-stained black dike(?) of plagioclase-bearing homblendite with } 1 \% \text { randomiy-orlented, disseminated } \\
\text { sulfides }\end{array}$ \\
\hline OORN330A & 431477 & 7131395 & Brecciated quartzite and schist \\
\hline 00RN331 & 431811 & 7131494 & Pyroxene skarn, skarnoid, and mineralized pyroxene hornfels with a little marble \\
\hline OORN332 & 431931 & 7131581 & Skarn \\
\hline OORN333 & 432133 & 7131636 & Sulfide-poor, coarse-grained, clinopyroxene skarn \\
\hline O0RN334 & $4333 \overline{13}$ & 7134650 & Aitered quartz porphyry \\
\hline OORN336 & 435419 & 7137735 & Orange-stained quartz porphyry dike \\
\hline 00RN337B & 437593 & 7140059 & Chalcedony + caicite + quartz; faulit breccia? \\
\hline OORN33BA & 437415 & 7139871 & Chalcedony veinlets \\
\hline OORN4B0A & 471097 & 7102407 & $\begin{array}{l}\text { Orange-stained orthogneiss with disseminated- and vein-style quartz-carbonate-pyrite-sericite alteralion and } \\
\text { disseminated secondary? biotite + albite? }\end{array}$ \\
\hline
\end{tabular}


Table I. Location and description of rock samples collected in the Eagle quadrangle for trace element geochemical analyses.

\begin{tabular}{|c|c|c|c|}
\hline Sample & UTME] & UTMN & Description \\
\hline 00RN515A & $4 \overline{71171}$ & 7103609 & Fault zone with brecciation, quartz, chaicedony, and iron-oxide \\
\hline 00RN515C & $471 \uparrow \overline{71}$ & $71036 \overline{09}$ & Amphibolite with up to $10 \%$ interstitial pyrite \\
\hline DORN523B & 462027 & $717 \overline{7204}$ & Skarn from skarn vein approximately $0.5 \mathrm{~m}$ thick composed of garnel, pyroxene, quartz, and pyrrhotile \\
\hline 00RN533B & $4 \overline{83522}$ & $7+13800$ & Quart + K-feldspar vein or pegmatite? dike \\
\hline 00RN560 & 484009 & 7115772 & Brecciated quartzite and quartz vein material; possible fault zone \\
\hline 00WM151 & 455570 & $71231 \overline{46}$ & Orange-, ye!low- and red-stained orthogneiss? \\
\hline $00 \mathrm{WM} 206$ & $4610 \overline{85}$ & $71007 \overline{51}$ & Gray brecciated limestone with calcite veins \\
\hline 00 WM210 & $\overline{4612 \overline{97}}$ & 7100377 & Green chloritic phyllite \\
\hline $00 \mathrm{WM} 269 \mathrm{~B}$ & 473820 & 7108705 & Brecciated quartzite \\
\hline$\overline{0} 0 \mathrm{WM} 369 \overline{\mathrm{B}}$ & $\overline{4835 \overline{43}}$ & 7119378 & Green-turquoise colored quartzite \\
\hline
\end{tabular}


Table 2. Concentration of trace elements in rock samples collected in the Eagle quadrangle. Note: --- = not analyzed.

\begin{tabular}{|c|c|c|c|c|c|c|c|c|c|c|c|c|c|c|c|c|c|c|c|}
\hline SAMPLE & $\mathrm{Au}$ & $\mathrm{Au}$ & $\mathrm{Ag}$ & $\mathrm{Al}$ & As & $\mathrm{B}$ & $\mathrm{Ba}$ & Be & $\mathrm{Bj}$ & $\mathrm{Ca}$ & $\mathrm{Cd}$ & $\overline{\mathrm{CO}}$ & $\overline{\mathrm{Cr}}$ & $\mathrm{Cu}$ & $\mathrm{Fe}$ & $\mathrm{Ga}$ & $\mathrm{Hg}$ & $K$ & La \\
\hline & $\mathrm{ppb}$ & oz/ton & ppm & $\%$ & ppm & ppm & ppm & ppm & ppm & $\%$ & ppm & ppm & ppm & ppm & $\%$ & $\mathrm{ppm}$ & ppm: & $\%$ & $\mathrm{ppm}$ \\
\hline 0005001 & $<5$ & --- & $<0.2$ & 0.51 & 6 & $<10$ & 70 & $<0.5$ & $<2$ & 0.25 & $<0.5$ & 6 & 167 & 31 & 1.00 & $<10$ & $<1$ & 0.09 & $<10$ \\
\hline 0005005 & $<5$ & 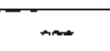 & 0.6 & 1.00 & 40 & $<10$ & 120 & $<\overline{0.5}$ & $<2$ & 0.07 & 2.5 & 5 & 87 & 9 & 2.95 & $<10$ & $<1$ & 0.19 & 10 \\
\hline $00 D S 010 A$ & $<5$ & $\cdots$ & 0.2 & 0.15 & $<2$ & $<10$ & 100 & $<\overline{0.5}$ & $<2$ & $>15.00$ & $<0.5$ & $<1$ & 8 & 1 & 0.36 & $<10$ & $<1$ & 0.01 & 10 \\
\hline ODDS010C & $<5$ & -- & $<0.2$ & 0.50 & $<2$ & $<10$ & 50 & $<0.5$ & $<2$ & 0.35 & $<0.5$ & $<1$ & 44 & 3 & 0.75 & $<10$ & $<1$ & 0.06 & $<10$ \\
\hline 00DS026B & $<5$ & $m$ & $<0.2$ & 0.48 & $<2$ & $<10$ & 60 & $\overline{0.5}$ & $<2$ & 0.35 & $<0.5$ & $<1$ & 102 & $<1$ & 0.43 & $<10$ & $<1$ & 0.11 & $<10$ \\
\hline $00 \mathrm{DS} 032 \mathrm{~B}$ & $<5$ & $\cdots$ & $<0.2$ & 0.67 & $<2$ & $<10$ & 190 & $<0.5$ & $<2$ & 0.08 & $<0.5$ & 1 & 102 & 44 & 1.79 & $<10$ & $<1$ & 0.24 & 10 \\
\hline $00 D S 039$ & $<5$ & $=$ & $<0.2$ & 0.37 & $<2$ & $<10$ & 10 & $<\overline{0.5}$ & $<2$ & $>15.00$ & $<0.5$ & 3 & 17 & 7 & 0.45 & $<10$ & 1 & 0.05 & $<10$ \\
\hline 00050458 & $<5$ & - & $<0.2$ & 0.46 & $<2$ & $<10$ & 120 & 0.5 & $<2$ & 8.73 & $<0.5$ & 6 & 20 & 32 & 1.01 & $<10$ & $<1$ & 0.04 & $<10$ \\
\hline OODS048A & $<5$ & $m$ & $<0.2$ & 1.98 & $<2$ & $<10$ & 150 & 0.5 & $<2$ & 3.18 & 4.5 & 9 & $\overline{15}$ & 18 & 5.48 & 10 & $<1$ & 0.34 & 10 \\
\hline $00 \mathrm{DS} 063 \mathrm{~A}$ & $<5$ & - & $<0.2$ & 0.56 & $<2$ & $<10$ & 120 & 0.5 & $<\overline{2}$ & 5.61 & $0 . \overline{5}$ & $\uparrow 2$ & 25 & 16 & 2.20 & $<10$ & $<1$ & 0.05 & 10 \\
\hline 00DS063B & $<5$ & - & $<0.2$ & $<0.01$ & $<2$ & $<10$ & 590 & $<0.5$ & $<2$ & $>15.00$ & $<0.5$ & $<1$ & 3 & $<1$ & 0.03 & $<10$ & 1 & $<0.01$ & $<10$ \\
\hline 00DS064 & $<5$ & - & $<0.2$ & 0.55 & $<2$ & $<10$ & 40 & 0.5 & $<2$ & 2.62 & $<0.5$ & $<1$ & 20 & $<1$ & 0.77 & $<10$ & $<1$ & 0.02 & 10 \\
\hline $0005070 \mathrm{~A}$ & $<5$ & - & $<0.2$ & 1.05 & $<2$ & $<10$ & 60 & $<0.5$ & $<2$ & 0.47 & $<0.5$ & 5 & 45 & 6 & 1.76 & $<10$ & $<1$ & 0.13 & $<10$ \\
\hline 00DS074 & $<5$ & $\cdots$ & $<0.2$ & 0.19 & $<2$ & $<10$ & 10 & $<\overline{0.5}$ & $<2$ & 9.42 & $<0.5$ & 1 & 61 & 6 & 0.37 & $<10$ & $<1$ & 0.04 & $<10$ \\
\hline 00DS075 & $<5$ & $\cdots$ & 0.2 & $<0.01$ & $<2$ & $<10$ & $<10$ & $<0.5$ & $<2$ & $>15.00$ & $<0.5$ & $<1$ & 1 & $<1$ & 0.03 & $<10$ & $<1$ & $<0.01$ & $<10$ \\
\hline 00DS079A & $<5$ & -- & $<0.2$ & 0.01 & $<2$ & $<10$ & $<10$ & $<0.5$ & $<2$ & 12.85 & $<0.5$ & $<1$ & 9 & $<1$ & 0.12 & $<1 \overline{0}$ & $<1$ & $<\overline{0.01}$ & $<10$ \\
\hline $0005079 \mathrm{~B}$ & $<5$ & -- & $\angle 0.2$ & 0.30 & $<2$ & $<10$ & 50 & $<0.5$ & $<2$ & 0.24 & $<0.5$ & 1 & 142 & 6 & 0.82 & $<10$ & $<1$ & 0.09 & $<10$ \\
\hline $000 S 081$ & $<5$ & $\cdots$ & $<0.2$ & 1.75 & 2 & $<10$ & 150 & 0.5 & $<2$ & 0.92 & 2 & 15 & 70 & 24 & 3.68 & $<10$ & $<1$ & 0.16 & 10 \\
\hline 0005085 & $<5$ & $\cdots$ & $<0.2$ & 1.26 & 2 & $<10$ & 60 & $<\overline{0.5}$ & $<2$ & 0.63 & 0.5 & 9 & 148 & 18 & 2.15 & $<10$ & $<1$ & 0.16 & 10 \\
\hline 00DS092A & $<5$ & $\cdots$ & $<0.2$ & 0.14 & 14 & $<10$ & 10 & $<0.5$ & $<2$ & 0.02 & $<0.5$ & 2 & 173 & 3 & 0.74 & $<10$ & $<1$ & 0.02 & $<10$ \\
\hline $00 D S 103 \mathrm{~A}$ & 10 & -- & $\overline{1}$ & 2.74 & 32 & $<10$ & 100 & $<0.5$ & $<2$ & 1.87 & $<0.5$ & 16 & $\overline{6} 2$ & 150 & 4.18 & 10 & $<1$ & 0.09 & $<10$ \\
\hline ODDS110A & 5 & -- & 0.2 & 0.84 & 6 & $<10$ & 100 & $<0.5$ & $<2$ & 5.74 & $<0.5$ & 10 & 27 & 15 & 3.28 & $<10$ & $<1$ & 0.21 & 10 \\
\hline OODS135A & 190 & -- & $\overline{0.2}$ & 0.13 & $<2$ & $<10$ & 1470 & $<0.5$ & $<2$ & 9.60 & $<0.5$ & 7 & 40 & 8 & 5.41 & $<1 \overline{0}$ & $<1$ & $0 . \overline{08}$ & 10 \\
\hline OODS135C & 465 & - & 0.4 & 0.80 & $<2$ & $<10$ & 560 & $<0.5$ & $<2$ & 3.23 & $<0.5$ & 6 & 14 & 19 & 2.05 & $<10$ & $<1$ & 0.16 & 10 \\
\hline OODS136A & $>10000$ & 0.693 & 6.6 & 0.23 & $<2$ & $<10$ & 180 & $<0.5$ & $<2$ & 0.24 & $<0.5$ & 7 & 85 & 3 & 1.41 & $<10$ & $<1$ & 0.13 & $<10$ \\
\hline $0005145 \mathrm{~B}$ & $<5$ & $\cdots$ & $<0.2$ & 0.46 & $<2$ & $<10$ & 10 & $<0.5$ & $<2$ & 0.88 & $<0.5$ & 10 & 134 & 1 & 1.38 & $<1 \overline{0}$ & $<1$ & 0.02 & $<10$ \\
\hline $00 \bar{D} 14 \overline{7 B}$ & $<5$ & - & $<0.2$ & 1.90 & $<2$ & $<10$ & 40 & $<0.5$ & $<2$ & 0.40 & $<0.5$ & 17 & 56 & 24 & 5.21 & $<10$ & $<1$ & 0.07 & $<10$ \\
\hline $00 \mathrm{DS160C}$ & $<5$ & - & 0.8 & 1.08 & $<2$ & $<10$ & 20 & 0.5 & $<2$ & $>15.00$ & $<0.5$ & 2 & 4 & $<1$ & $\overline{0.68}$ & $<10$ & $<1$ & 0.08 & 10 \\
\hline 00DS165 & $<5$ & $\cdots$ & $<0.2$ & 0.50 & $<2$ & $<10$ & 50 & $<0.5$ & $<2$ & $\overline{2.02}$ & $<0.5$ & 3 & 126 & 5 & 0.97 & $<10$ & $<1$ & 0.09 & $<10$ \\
\hline OODS168B & $<5$ & $=$ & $<0.2$ & 4.10 & $<2$ & $<10$ & 80 & 1 & $<2$ & 2.96 & 2 & 75 & 6 & 2 & $>15.00$ & $<10^{\circ}$ & $<1$ & 0.19 & $<10$ \\
\hline $000 \$ 186 \mathrm{~A}$ & 20 & -- & $<0.2$ & 0.97 & $<2$ & $<10$ & 90 & 0.5 & $<2$ & 1.76 & $<0.5$ & $<1$ & 96 & $<1$ & 0.56 & $<10$ & $<1$ & 0.03 & $<10$ \\
\hline 00DS187B & $<5$ & - & $<0.2$ & 1.10 & $<2$ & $<10$ & 150 & $<0.5$ & $<2$ & 0.41 & $<0.5$ & 5 & 139 & 50 & 1.51 & $<10$ & $<1$ & 0.31 & $<10$ \\
\hline OODS200B & 5 & - & 0.2 & 1.03 & $<2$ & $<10$ & 40 & $<0.5$ & $<2$ & 12.40 & $<0.5$ & $<1$ & 31 & 4 & 0.24 & $<1 \overline{0}$ & $<1$ & 0.04 & $<10$ \\
\hline 00DS215 & 10 & $\cdots$ & $<0.2$ & 0.82 & $<2$ & $<10$ & 90 & $<0.5$ & $<2$ & 0.42 & $<0.5$ & 6 & 100 & 23 & 1.89 & $<10$ & $<1$ & 0.16 & $<10$ \\
\hline OODS217 & $<5$ & 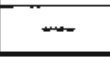 & $<0.2$ & 2.02 & $<2$ & $<10$ & 90 & $<\overline{0.5}$ & $<2$ & 1.50 & $<\overline{5}$ & 11 & 43 & 33 & 3.03 & $<10$ & $<1$ & 0.13 & $<10$ \\
\hline
\end{tabular}


Table 2. Concentration of trace elements in rock samples collected in the Eagle quadrangle. Note: --- = not analyzed.

\begin{tabular}{|c|c|c|c|c|c|c|c|c|c|c|c|c|c|c|c|c|c|c|c|}
\hline SAMPLE & $\mathrm{Au}$ & $\mathrm{Au}$ & $\mathrm{Ag}$ & Al & As & B & $\overline{B a}$ & $\mathrm{Be}$ & $\mathrm{Bi}$ & $\mathrm{Ca}$ & $\mathrm{Cd}$ & Co & $\mathrm{Cr}$ & $\mathrm{Cu}$ & $\mathrm{Fe}$ & $\mathrm{Ga}$ & $\mathrm{Hg}$ & $\mathrm{K}$ & Là \\
\hline & $\mathrm{ppb}$ & oz/ton & $\mathrm{ppm}$ & $\%$ & $\mathrm{ppm}$ & ppm & ppm & $\mathrm{ppm}$ & $\mathrm{ppm}$ & $\%$ & ppm & $\mathrm{ppm}$ & $\mathrm{ppm}$ & ppm & $\%$ & $\mathrm{ppm}$ & $\mathrm{ppm}$ & $\%$ & $\mathrm{ppm}$ \\
\hline $00 \mathrm{JG} 042 \mathrm{C}-6 / 30$ & 5 & - & 0.2 & 0.31 & 2 & $<10$ & 80 & $<0.5$ & $<2$ & 0.05 & $<0.5$ & $<1$ & 77 & 2 & 0.68 & $<10$ & $<1$ & 0.14 & $<10$ \\
\hline $50 \mathrm{~A}-6 / 44$ & $<5$ & -- & $<0.2$ & 0.27 & $<2$ & $<10$ & 280 & $<0 . \overline{5}$ & $<2$ & 0.54 & $<0.5$ & $<1$ & 52 & 2 & 0.32 & $<10$ & $<1$ & .07 & 10 \\
\hline $50 \mathrm{~B}-6 / 14$ & $<\dot{5}$ & $+m$ & $<0.2$ & 1.26 & $<2$ & $<10$ & 330 & $<0.5$ & $<2$ & 0.80 & 40.5 & 3 & 59 & 4 & 2.15 & $<10$ & $<1$ & 0.46 & 10 \\
\hline $63-6 / 16$ & $<5$ & $-\cdots$ & $<0,2$ & 1.02 & $<2$ & $<10$ & 30 & $<0.5$ & $<2$ & 1.67 & $<0.5$ & 1 & 31 & 1 & 0.99 & $<10$ & $<1$ & 0.06 & $\leq 10$ \\
\hline $63 \times-7 / 1$ & $<5$ & $\cdots$ & $<0.2$ & 2.31 & $<2$ & $<10$ & 50 & $<0.5$ & $<2$ & 2.36 & $<0.5$ & 27 & 7 & 7 & 7.33 & 10 & $<1$ & 0.05 & $<10$ \\
\hline $57-7 / 2$ & 5 & -- & $<0.2$ & 1.49 & $<2$ & $<10$ & 420 & $<0.5$ & $<2$ & 2.06 & 0.5 & 6 & 24 & 7 & 3.16 & $<10$ & $<1$ & 0.59 & $<10$ \\
\hline$2 - 6 \longdiv { 1 9 }$ & $<5$ & - & $<0.2$ & 1.31 & $<2$ & $<10$ & 160 & $<0.5$ & $<2$ & 0.70 & $<0.5$ & 5 & 78 & 12 & 2.52 & $<10$ & $<1$ & 0.24 & 10 \\
\hline $3 \mathrm{~A}-7 / 2$ & $<5$ & - & $<0.2$ & 0.93 & 2 & $<10$ & 40 & $<0.5$ & $\angle 2$ & 0.43 & $<0.5$ & 6 & 78 & 12 & 2.02 & $<10$ & $<1$ & 0.07 & $<10$ \\
\hline $7-6 / 19$ & $<\overline{5}$ & - & 0.2 & 1.70 & 10 & $<10$ & 20 & $<0.5$ & $<2$ & 1.33 & $<0.5$ & 20 & 71 & 55 & 4.37 & $<10$ & $<1$ & 0.13 & $<10$ \\
\hline $2-6 / 19$ & $<5$ & - & $<\overline{0.2}$ & 2.79 & $<2$ & $<10$ & 150 & $<0.5$ & $<2$ & 1.34 & $<0.5$ & 11 & 115 & 26 & 3.54 & $<10$ & $<1$ & $0 . \overline{47}$ & 10 \\
\hline $44-6 / 19$ & $<5$ & - & 0.6 & 0.43 & $<2$ & $<10$ & 30 & $<0.5$ & $<2$ & $>15.00$ & $<0.5$ & 2 & 30 & 4 & 0.74 & $<10^{-}$ & $<1$ & 0.24 & 10 \\
\hline $9 \mathrm{~B}-7 / 4$ & $<5$ & -- & $<0.2$ & 0.24 & $<2$ & $<10$ & 40 & $<0.5$ & $<2$ & 0.08 & $<0,5$ & $<1$ & 44 & 2 & 1.01 & $<10$ & $<1$ & 0.2 & 20 \\
\hline $3 \mathrm{~B}-7 / 9$ & $<5$ & -- & $<0.2$ & 0.24 & $<2$ & $<10$ & 170 & $<0.5$ & $<2$ & 0.08 & $<0.5$ & 1 & 47 & 3 & 0.30 & $<10$ & $<1$ & 0.09 & $<10$ \\
\hline $7 / 9$ & $<5$ & --- & 1.4 & 2.13 & $<2$ & $<10$ & 110 & $<0 . \overline{5}$ & $<2$ & 2.15 & $<0.5$ & 32 & 62 & 103 & 3.95 & $<10$ & $<1$ & 0.09 & $<10$ \\
\hline $6-6 / 23$ & $<5$ & $\cdots$ & $<0.2$ & 1.41 & $<2$ & $<10$ & 60 & $<0.5$ & $<2$ & 1.01 & $<0.5$ & 7 & 58 & 5 & 2.45 & $<10$ & $<1$ & 0.17 & $<10$ \\
\hline $7-6 / 23$ & $<5$ & --- & $<0.2$ & 1.09 & 52 & $<10$ & 50 & $<0.5$ & $<2$ & 0.72 & $<0.5$ & 6 & 37 & $\overline{7}$ & 2.09 & $<10$ & $<1$ & 0.33 & $<10$ \\
\hline $8.6 / 23$ & $<5$ & $\cdots$ & $<0.2$ & 0.90 & $<2$ & $<10$ & 50 & $<0.5$ & $<2$ & 0.74 & $<0.5$ & 5 & 24 & 3 & 1.46 & $<10$ & $<1$ & 0.15 & $<1 \overline{0}$ \\
\hline $9-6 / 23$ & $<5$ & - & $<0.2$ & 0.99 & $<2$ & $<10$ & 90 & $<0.5$ & $<2$ & 0.67 & $<0.5$ & 5 & 29 & $<1$ & 1.40 & $<10$ & $<1$ & 0.16 & $<10$ \\
\hline $0-\overline{6 / 23}$ & $<5$ & - & $<\overline{0.2}$ & 1.64 & $<2$ & $<10$ & 380 & $<0.5$ & $<2$ & 0.78 & $<0.5$ & 13 & 63 & 7 & 2.60 & $<10$ & $<1$ & $0 . \overline{96}$ & $<10$ \\
\hline $5-6 / 25$ & $<\overline{5}$ & - & $<0.2$ & 0.25 & 2 & $<10$ & 220 & $<0.5$ & $<2$ & 0.33 & $<0.5$ & 5 & 137 & 36 & 0.76 & $<10$ & $<1$ & 0.05 & $<10$ \\
\hline COID\#1- & 10 & - & 0.8 & 0.16 & $<2$ & $<10$ & $<10$ & $<0.5$ & $<2$ & $>15.00$ & $<0.5$ & $<1$ & 10 & 7 & 0.17 & $<10$ & $<1$ & $<0.01$ & 10 \\
\hline KOID\#2-6/15 & $<5$ & 二 & $<0.2$ & 0.36 & $<2$ & $<10$ & 100 & $<0.5$ & $<2$ & 3.30 & $<0.5$ & 1 & 53 & 14 & 0.41 & $<10$ & $<1$ & 0.11 & $<10$ \\
\hline $11 \bar{A}$ & $1 \overline{5}$ & $\cdots$ & $<0,2$ & 0.57 & $<2$ & $<10$ & 50 & $<0 . \overline{5}$ & $<2$ & 0.12 & $<0.5$ & $\leq 1$ & 65 & 6 & 2.14 & $<10$ & $<1$ & 0.28 & $<10$ \\
\hline $38 \mathrm{~A}$ & $<5$ & - & $<0.2$ & 0.08 & $<2$ & $<10$ & $<10$ & $<0.5$ & $<2$ & 0.01 & $<0.5$ & $\overline{1}$ & $17 \overline{7}$ & 3 & 0.35 & $<10$ & $<1$ & $<0.01$ & $<10$ \\
\hline & $<5$ & -- & $<0.2$ & 1.19 & $<2$ & $<10$ & 250 & $<0.5$ & $\leq 2$ & 0.68 & $<0.5$ & 4 & 93 & 35 & 2.02 & $<10$ & $<1$ & 0.76 & $<10$ \\
\hline $94 \mathrm{~B}$ & $<5$ & - & $0 . \overline{4}$ & 1.28 & $<2$ & $<10$ & 190 & $<0.5$ & $<2$ & 0.32 & $<0.5$ & 11 & 85 & $\{66$ & 2.87 & $<10$ & $<1$ & 0.75 & $<10$ \\
\hline $\mathrm{V} 101 \mathrm{~A}$ & $1 \overline{10}$ & 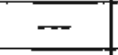 & $<\overline{2}$ & $0.8 \overline{8}$ & $\overline{<2}$ & $<10$ & 40 & $<0.5$ & $<2$ & 1.07 & $<0.5$ & 3 & 42 & 1 & 1.03 & $<10$ & $<1$ & 0.14 & $<10$ \\
\hline Vino1C & 85 & $\cdots$ & $<0.2$ & 0.23 & 2 & $<10$ & 150 & $<0.5$ & $<2$ & 0.04 & $<0.5$ & 3 & 111 & 3 & 1.02 & $<10$ & $<1$ & 0.09 & $<10$ \\
\hline 104 & 10 & - & $<0.2$ & 0.54 & 8 & $<10$ & 270 & 1 & $<2$ & 4.96 & $<0.5$ & 8 & 30 & 4 & 3.65 & $<10$ & $<1$ & $0 . \overline{28}$ & 10 \\
\hline 111 & $66 \overline{5}$ & --- & $<0.2$ & 0.56 & 2 & $<10$ & 240 & $<0.5$ & $<2$ & 0.30 & $<0.5$ & 4 & 95 & 2 & 1.70 & $<10$ & $\leq 1$ & 0.11 & 10 \\
\hline V112 & 10 & 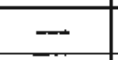 & $<0.2$ & 0.49 & $<2$ & $<10$ & 640 & 0.5 & $<2$ & 0.71 & $<0.5$ & $\overline{14}$ & $6 \overline{1}$ & 21 & 2.10 & $<10$ & $\overline{1}$ & 0.1 & 10 \\
\hline OOMBW130 & $<5$ & - & $<0.2$ & 0.15 & 46 & $\leq 10$ & 1750 & $<0.5$ & $<2$ & 0.02 & $<0.5$ & 3 & 148 & 12 & 0.82 & $<10$ & $<1$ & 0.05 & $<10$ \\
\hline OOMBW142B & $<5$ & $=$ & $<0.2$ & 0.34 & 24 & $<10$ & 40 & $<0.5$ & $<2$ & 0.03 & $<0.5$ & 7 & 150 & 93 & 4.14 & $<10$ & $<1$ & 0.11 & $<10$ \\
\hline N142C & $<5$ & $=$ & $<0.2$ & 0.29 & 6 & $<10$ & 110 & $<0.5$ & $<2$ & 0.04 & $<0.5$ & 2 & $6 \overline{7}$ & 12 & 0.39 & $<10$ & $<1$ & 0.21 & $<10$ \\
\hline OOMBW180 & $<5$ & - & $<0.2$ & 1.73 & $<2$ & $<10$ & 10 & $<0.5$ & $<2$ & 2.73 & $<0.5$ & 6 & $3 \overline{8}$ & 2 & 0.67 & $<10$ & $<1$ & 0.01 & $<10$ \\
\hline
\end{tabular}


Table 2. Concentration of trace elements in rock samples collected in the Eagle quadrangle. Note: --- = not analyzed.

\begin{tabular}{|c|c|c|c|c|c|c|c|c|c|c|c|c|c|c|c|c|c|c|c|}
\hline SAMPLE & $\mathrm{Au}$ & $\mathrm{Au}$ & $\mathrm{Ag}$ & Al & As & $\bar{B}$ & $\mathrm{Ba}$ & $\mathrm{Be}$ & $\mathrm{Bi}$ & $\mathrm{Ca}$ & $\overline{C d}$ & $\overline{\mathrm{Co}}$ & $\mathrm{Cr}$ & $\mathrm{Cu}$ & $\mathrm{Fe}$ & $\mathrm{Ga}$ & $\mathrm{Hg}$ & $K$ & $\mathrm{La}$ \\
\hline & $p \overline{p b}$ & ozfton & ppm & $\%$ & $\mathrm{ppm}$ & $p p m$ & ppm & ppm & ppm & $\%$ & ppm & ppm & ppm & ppm & $\%$ & ppm & ppm & $\%$ & $\mathrm{ppm}$ \\
\hline OOMBW187日 & $<5$ & - & $<0.2$ & 0.27 & 2 & $<10$ & 210 & $<0.5$ & $<2$ & 0.04 & $<0.5$ & $<1$ & 61 & 17 & 0.43 & $<10$ & $<1$ & 0.18 & $<10$ \\
\hline 00MBW190 & 65 & - & $<0.2$ & 0.35 & $<2$ & $\angle 10$ & 90 & $<0.5$ & 4 & 0.82 & 0.5 & 1 & 40 & 13 & 0.75 & $<10$ & $<1$ & 0.01 & $<10$ \\
\hline 00MBW19\} & $<5$ & - & 1 & 2.49 & $<2$ & $<10$ & 400 & 0.5 & 2 & 1.62 & 3.5 & 3 & 164 & 26 & 1.06 & $<10$ & $<1$ & 0.07 & 10 \\
\hline O0MBW192 & $<5$ & - & 6.6 & $2 . \overline{74}$ & $<2$ & $<10$ & 80 & $<0.5$ & $\overrightarrow{14}$ & 1.81 & $10^{-}$ & $<1$ & 65 & 15 & 0.71 & $<10$ & $<1$ & 0.03 & $<10$ \\
\hline OOMBW193 & $<5$ & - & $<0.2$ & 0.26 & $<2$ & $<10$ & 430 & $<0.5$ & $<2$ & 0.04 & $<0.5$ & 1 & 93 & 5 & 0.26 & $\angle 10$ & $<1$ & 0.14 & $<10$ \\
\hline OOMBW200 & $<\overline{5}$ & - & $<0.2$ & 1.50 & $<2$ & $<10$ & 100 & $<0.5$ & $<2$ & 2.36 & $<0.5$ & $\overline{8}$ & 48 & 5 & 2.17 & $<10$ & $<1$ & $\overline{0 . \overline{12}}$ & $<10$ \\
\hline DOMBW215B & $<5$ & - & $<0.2$ & 0.92 & $<2$ & $<10$ & 60 & 0.5 & $<2$ & 0.42 & 3 & 4 & 130 & $\overline{22}$ & 1.50 & $<10$ & $<1$ & $\overline{0.15}$ & 10 \\
\hline OOMBW217 & $<5$ & -- & $<0.2$ & 0.91 & $<2$ & $<10$ & 80 & 2.5 & $<2$ & 1.56 & 3.5 & 1 & 116 & 6 & 1.20 & $<10$ & $<1$ & 0.05 & $<10$ \\
\hline OOMBW224 & $<5$ & $\cdots$ & $<0.2$ & 0.27 & $<2$ & $\angle 10$ & 2240 & $<0.5$ & $<2$ & 0.06 & $<0.5$ & $<1$ & 84 & 6 & 0.98 & $<10$ & $<1$ & 0.11 & 10 \\
\hline 00MBW230 & $<\overline{5}$ & $\overline{--}$ & $<0.2$ & $\overline{0.41}$ & $<2$ & $<10$ & 60 & $<0 . \overline{5}$ & $<2$ & 0.18 & $<0.5$ & 2 & 222 & 9 & 1.07 & $<10$ & $<1$ & $0 . \overline{16}$ & $<1 \overline{0}$ \\
\hline O0MBW250 & $<5$ & -- & $<\overline{0.2}$ & 0.68 & $<2$ & $<10$ & 140 & $<0.5$ & $<2$ & 0.07 & $<0.5$ & 3 & 92 & 8 & 1.07 & $<10$ & $<1$ & $0 . \overline{35}$ & $20^{-}$ \\
\hline OOMBW251 & $<5$ & $\cdots$ & $<0.2$ & 0.08 & $<2$ & $<10$ & 20 & $<0.5$ & $<2$ & 0.02 & $<0.5$ & $<1$ & 202 & 1 & 0.33 & $<10$ & $<1$ & 0.06 & $<10$ \\
\hline 00MBW264 & $<5$ & $-\cdots$ & $<0.2$ & 0.26 & 2 & $<10$ & 310 & $<0.5$ & $<2$ & 0.03 & $<0.5$ & 7 & 144 & 11 & 0.84 & $<10$ & $<1$ & $0 . \overline{0} 1$ & $<10$ \\
\hline 00MBW269 & $<5$ & $-\cdots$ & $<0.2$ & 0.54 & 10 & $<10$ & 30 & $<0,5$ & 2 & 0.04 & $<0.5$ & 7 & 130 & 24 & 1.51 & $<10$ & $<1$ & 0.04 & $<10$ \\
\hline OOMBW280 & $<5$ & --- & $<0.2$ & 2.90 & $<2$ & $<10$ & 370 & $<0.5$ & $<2$ & 0.36 & $<0.5$ & 32 & $6 \overline{0}$ & 19 & 5.79 & 90 & $<1$ & 0.39 & $<1 \overline{0}$ \\
\hline 00MBW281 & $<5$ & -- & $<0.2$ & 1.64 & 2 & $<10$ & 50 & $<0.5$ & $<2$ & 0.36 & $<0.5$ & 11 & 110 & 124 & 3.53 & $<10$ & $<1$ & 0.09 & 10 \\
\hline 00MBW306 & $<5$ & -- & 2.4 & 1.71 & 70 & $<10$ & 330 & $0 . \overline{5}$ & $<2$ & 0.09 & 0.5 & 16 & 63 & 35 & 5.14 & $\angle 10$ & $<1$ & 0.14 & $<10$ \\
\hline 00MBW307A & $<5$ & - & $<0.2$ & 1.85 & $<2$ & $<10$ & $\overline{50}$ & $<0.5$ & $<2$ & 1.47 & 0.5 & 13 & 62 & 4 & 3.59 & $<10$ & $<1$ & 0.07 & $<10$ \\
\hline $00 M B W 308 B$ & 5 & $\overline{-}$ & $\overline{7.6}$ & $1.4 \overline{8}$ & 54 & $<10$ & 130 & $<0.5$ & $<2$ & 0.11 & $<0.5$ & 14 & $12 \overline{8}$ & 198 & 3.12 & $<10$ & $<1$ & 0.11 & $<10$ \\
\hline DOM̈BW3108 & 85 & $\overline{-}$ & $\overline{6.6}$ & 0.37 & 48 & $<10$ & $4 \overline{0}$ & $<0.5$ & $<2$ & 0.03 & $<0.5$ & 1 & 126 & 52 & 0.76 & $<10$ & $<1$ & $0 . \overline{16}$ & $<10$ \\
\hline OOMBW311B & $<5$ & -- & 0.2 & 0.10 & 10 & $<10$ & 10 & $<0.5$ & $<2$ & $<0.01$ & $<0.5$ & $<1$ & $20 \overline{6}$ & 5 & 0.98 & $<10$ & $<1$ & 0.03 & $<10$ \\
\hline OOMBW321 & $<5$ & -- & $<0.2$ & 0.91 & 2 & $<10$ & 30 & $<0.5$ & $<2$ & 0.05 & 0.5 & 6 & 66 & 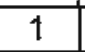 & 2.85 & $<10$ & $<1$ & $0 . \overline{05}$ & 10 \\
\hline OOMBW343 & $<5$ & - & $<0.2$ & 0.26 & $<2$ & $<10$ & 180 & $<0.5$ & $<2$ & 0.02 & $<0 . \overline{5}$ & $<1$ & 116 & 1 & 0.47 & $<10$ & $<1$ & 0.15 & $<10$ \\
\hline 00MBW351 & $<5$ & -- & $<\overline{0.2}$ & 1.20 & $<2$ & $<10$ & 200 & $<0.5$ & $<2$ & 0.67 & 1 & 8 & 99 & 18 & 2.15 & $<10$ & $<1$ & 0.25 & $<10$ \\
\hline $00 M B W 354$ & $<5$ & $\cdots$ & 0.2 & 0.31 & 2 & $<10$ & 130 & $<0.5$ & $<2$ & 0.07 & $<0 . \overline{5}$ & $<1$ & 120 & 2 & 0.38 & $<10$ & $<1$ & $0 . \overline{12}$ & 410 \\
\hline 00MBW355C & $<5$ & $\cdots$ & 0.2 & 0.11 & 6 & $<10$ & 20 & $<0.5$ & $<2$ & 0.07 & $<0.5$ & 2 & 207 & 27 & 0.93 & $\angle 10$ & $<1$ & 0.05 & $<10$ \\
\hline 00MBW356A & $<5$ & -- & $<\overline{0.2}$ & 0.18 & 10 & $<10$ & 250 & $<0.5$ & $<2$ & 0.27 & $<0.5$ & 3 & $13 \overline{3}$ & 4 & 0.49 & $<10$ & $<1$ & 0.1 & $<10$ \\
\hline $00 \mathrm{MBW} 356 \mathrm{~B}$ & $<5$ & -- & $<0.2$ & 0.35 & $<2$ & $<10$ & 90 & $<0.5$ & $<2$ & 0.36 & $<0.5$ & 1 & $7 \overline{6}$ & 1 & 0.48 & $<10$ & $<1$ & 0.11 & $<1 \overline{0}$ \\
\hline 00MBW384 & $<\overline{5}$ & 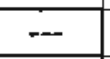 & $<0.2$ & 0.38 & $<2$ & $<10$ & 80 & $<0.5$ & $<2$ & 0.63 & $<0.5$ & 2 & 78 & 13 & 0.87 & $<10$ & $\leq 1$ & 0.08 & $<10$ \\
\hline OOMBW443 & $<5$ & - & $<0.2$ & 1.53 & $<2$ & $<10$ & 130 & 0.5 & $<2$ & 2.34 & $<0.5$ & 11 & 41 & 10 & 2.60 & $<10$ & $<1$ & 0.34 & 10 \\
\hline OOMBW460B & $<5$ & - & 0.2 & 1.98 & $<2$ & $<10$ & 370 & $<0.5$ & $<2$ & 0.68 & $<0.5$ & 18 & 47 & 67 & 5.51 & $<10$ & $<1$ & 0.78 & $<10$ \\
\hline 00MBW461A & $<5$ & - & $<0.2$ & 1.87 & $<2$ & $<10$ & 110 & $<0.5$ & $<2$ & 0.28 & $<\overline{0.5}$ & 13 & 11 & 5 & 4.58 & $<10$ & $<1$ & 0.21 & $<10$ \\
\hline O0MBW469 & $<5$ & --- & $<0.2$ & 0.50 & $<2$ & $<10$ & 180 & $<0.5$ & $<2$ & 0.08 & $<\overline{0.5}$ & 1 & 97 & 3 & 1.34 & $<10$ & $<1$ & 0.32 & $<1 \overline{0}$ \\
\hline DONBW513 & $<5$ & - & $<\overline{0.2}$ & 0.31 & $<2$ & $<10$ & 310 & $<0.5$ & $<2$ & 0.04 & $<0.5$ & $<1$ & $9 \overline{1}$ & 1 & 0.23 & $<10$ & $<1$ & 0.09 & $<10$ \\
\hline OOMBW523 & $<5$ & --- & $<0.2$ & 0.13 & $<2$ & $<10$ & 10 & $<0 . \overline{5}$ & $<2$ & 0.01 & $<0.5$ & $<1$ & 218 & 4 & 0.55 & $<10$ & $<1$ & $0 . \overline{05}$ & $<1 \overline{0}$ \\
\hline
\end{tabular}




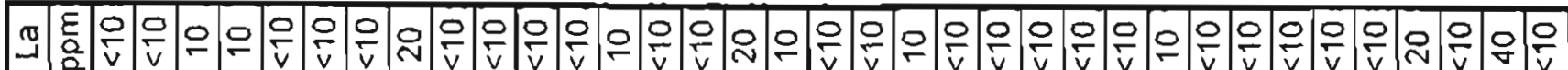
$x: 0$ F

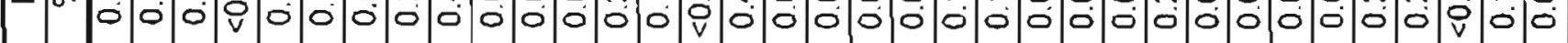

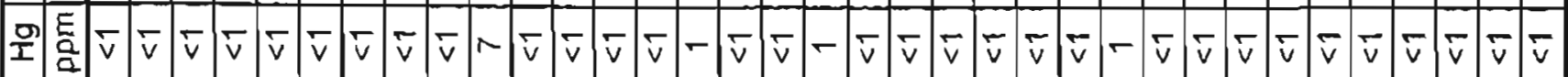

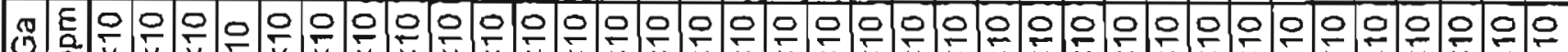

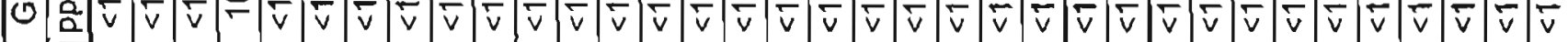

4்

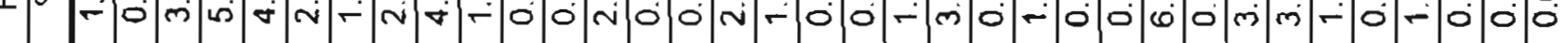

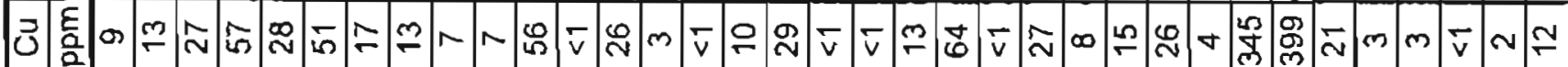

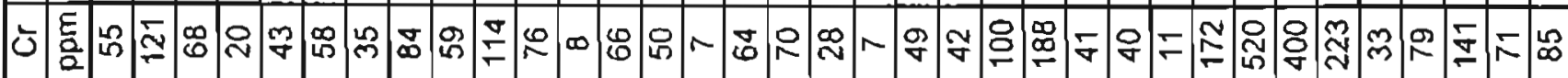
宁高至 - E

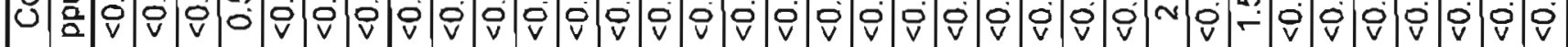
J

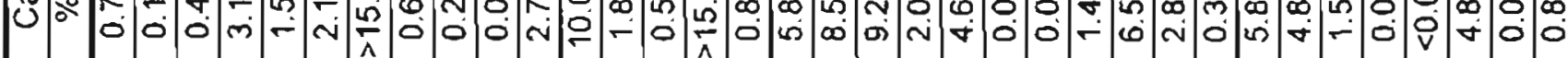

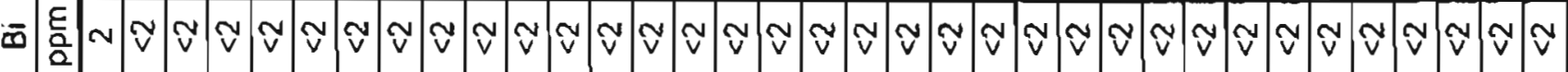

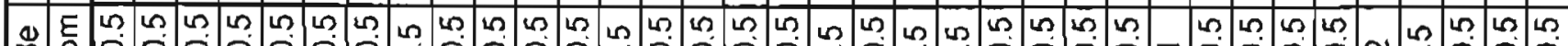

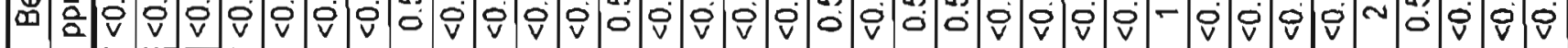
๓

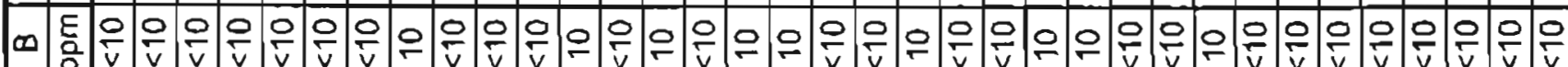

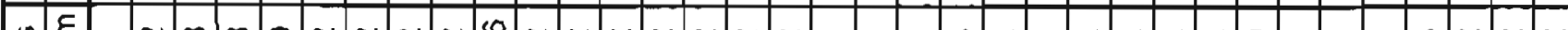

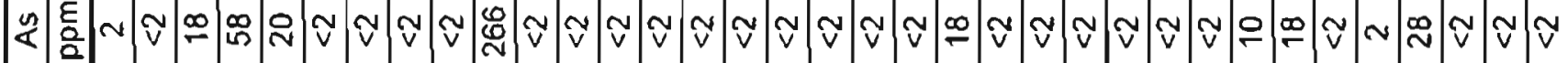
ব๐

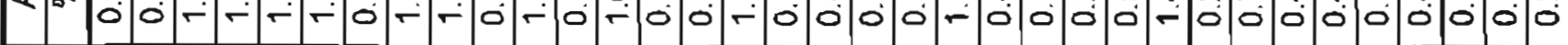

g E 年重 군

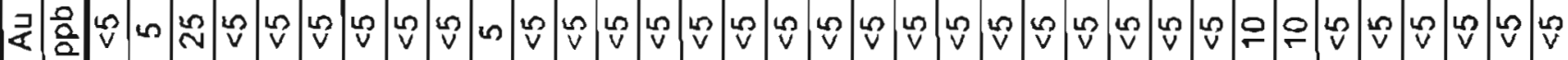

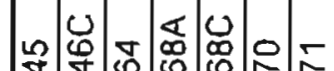

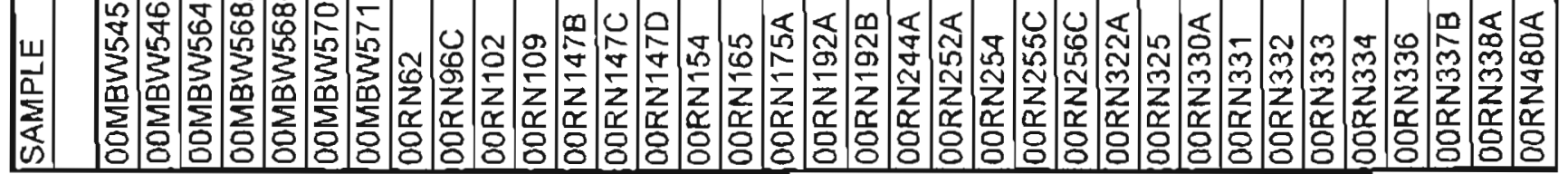


Table 2. Concentration of trace elements in rock samples collecled in the Eagle quadrangle. Note: --- = not analyzed.

\begin{tabular}{|c|c|c|c|c|c|c|c|c|c|c|c|c|c|c|c|c|c|c|c|}
\hline SAMPLE & $\mathrm{Au}$ & $\mathrm{Au}$ & $\mathrm{Ag}$ & $\mathrm{Al}$ & As & $\mathrm{B}$ & $\mathrm{Ba}$ & $\mathrm{Be}$ & $\mathrm{Bi}$ & $\mathrm{Ca}$ & $\mathrm{Cd}$ & $\mathrm{Co}$ & $\mathrm{Cr}$ & $\mathrm{Cu}$ & $\mathrm{Fe}$ & $\mathrm{Ga}$ & $\mathrm{Hg}$ & $\bar{K}$ & La \\
\hline & $\mathrm{ppb}$ & oz/ton & ppm & $\%$ & ppm & ppm & ppm & ppm & $\mathrm{ppm}$ & $\%$ & ppm & ppm & ppm & ppm & $\%$ & $\mathrm{ppm}$ & ppm & $\%$ & $\mathrm{ppm}$ \\
\hline OORN481A & $<5$ & - & 02 & 0.45 & 48 & $<10$ & 30 & 1 & $<2$ & 3.52 & 1 & 35 & 142 & 91 & 4.84 & $<10$ & $<1$ & 0.07 & $\angle 10$ \\
\hline OORN481B & $<5$ & - & $\overline{0.2}$ & 0.38 & 12 & $<\overline{10}$ & $7 \overline{70}$ & $<0.5$ & $<2$ & $0 . \overline{87}$ & $<0.5$ & 6 & 147 & 88 & 1.72 & $<10^{-}$ & $<1$ & 0.08 & $<10$ \\
\hline OORN493A & $<5$ & - & 0.2 & 1.83 & $<2$ & $<10$ & 70 & $<0.5$ & $<2$ & 2.45 & $<0.5$ & 31 & 66 & 44 & 4.82 & $<10$ & $<1$ & 0.54 & $<10$ \\
\hline O0RN515A & 10 & $\cdots$ & 0.2 & 0.41 & 12 & $<10$ & 150 & $<0.5$ & $<2$ & 0.08 & $<0.5$ & 3 & 117 & 15 & 1.84 & $<10$ & $<1$ & 0.29 & $<10$ \\
\hline OORN515B & $<5$ & - & $<0.2$ & 0.11 & 2 & $<10$ & 290 & $<\overline{0} .5$ & $<2$ & 0.03 & $<0.5$ & $<1$ & 108 & 1 & 1.00 & $<10$ & $<1$ & 0.17 & 610 \\
\hline OORN515C & 5 & $\cdots$ & 0.8 & 1.83 & $<2$ & $<10$ & 10 & $<0.5$ & $<2$ & 1.30 & $<0.5$ & 29 & 45 & 164 & 9.44 & $<10$ & $<1$ & 0.66 & $<10$ \\
\hline OORN523A & 15 & - & $<0.2$ & 0.76 & 18 & $<\overline{10}$ & 50 & $<0.5$ & $<2$ & $1 . \overline{39}$ & $<0.5$ & 14 & 138 & 54 & 2.35 & $<10$ & $<1$ & $\overline{0.09}$ & 10 \\
\hline $00 \mathrm{RN} 523 \mathrm{~B}$ & $<5$ & - & 0.4 & 0.33 & 6 & $<10$ & 10 & $<0.5$ & $<2$ & $\overline{5.45}$ & $<0.5$ & 10 & 52 & 53 & 2.13 & $<10$ & $<1$ & $<0.01$ & $<10$ \\
\hline OORN533B & $<5$ & -- & $<0.2$ & 0.27 & $<2$ & $<\overline{10}$ & 150 & $<0.5$ & $<2$ & $0 . \overline{03}$ & $<0.5$ & 2 & 160 & 6 & 0.63 & $<10$ & $<1$ & 0.22 & $<10$ \\
\hline 00RN560 & $<5$ & - & $<0.2$ & 0.14 & 14 & $<10$ & 50 & $<0.5$ & $<2$ & 0.21 & $<0.5$ & 2 & 94 & 13 & 0.58 & $<10$ & $<1$ & 0.04 & $<10$ \\
\hline 00 WM 133 & $<5$ & - & $<\overline{0.2}$ & 1.71 & $<2$ & $<10$ & 610 & $<0.5$ & $<2$ & 1.54 & $<0.5$ & 15 & 46 & 17 & 2.68 & $<10$ & $<1$ & 0.72 & $<10$ \\
\hline $00 \mathrm{WM} 144 \mathrm{C}$ & 5 & $\cdots$ & 0.2 & 2.29 & $<2$ & $<10$ & 110 & $<0.5$ & $<2$ & 0.31 & $<0.5$ & 13 & 46 & $4 \overline{43}$ & 4.92 & 10 & $<1$ & 0.16 & $<10$ \\
\hline 00 WM151 & 5 & -- & $<0.2$ & 0.48 & $<2$ & $<10$ & 130 & 0.5 & $<2$ & 0.04 & $<0.5$ & 3 & 26 & 38 & 6.00 & $<10$ & $<1$ & 0.34 & $<10$ \\
\hline $00 \mathrm{WM} \overline{206}$ & $<5$ & $\cdots$ & 1 & 0.05 & $<2$ & $<10$ & $<10$ & $<0.5$ & $<2$ & $>15.00$ & $<0 . \overline{5}$ & $<1$ & 2 & $\overline{7}$ & 0.13 & $<10$ & $<1$ & 0.01 & $<10$ \\
\hline OOWM210 & $<5$ & --- & $\overline{\angle 0.2}$ & 2.79 & $<2$ & $<10$ & $270^{\circ}$ & $<0.5$ & $<2$ & 2.75 & $<0.5$ & 37 & 236 & 62 & $6 . \overline{08}$ & 10 & $<1$ & 1.28 & $<10$ \\
\hline 00WM269B & $<5$ & - & 0.6 & 0.08 & 6 & $<10$ & 560 & $<0.5$ & $<2$ & 0.04 & $<0.5$ & $<1$ & 131 & 4 & 0.54 & $<10$ & $<1$ & 0.07 & $<10$ \\
\hline $00 \mathrm{WM} 369 \mathrm{~B}$ & $<5$ & $=$ & 3 & 0.32 & 4 & $<10$ & 210 & $<0.5$ & $<2$ & $0 . \overline{50}$ & 17 & $<1$ & 154 & 9 & 0.79 & $<10$ & $<1$ & 0.14 & 10 \\
\hline
\end{tabular}




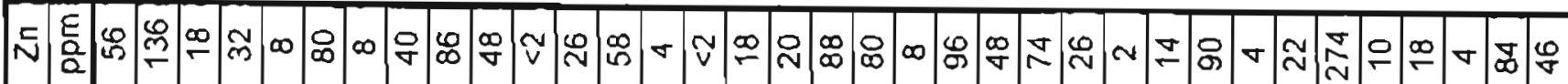

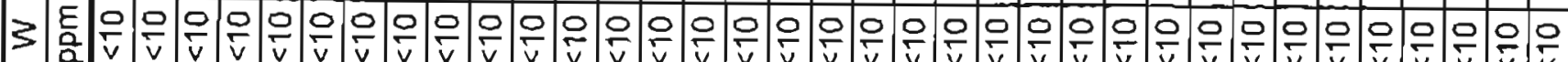

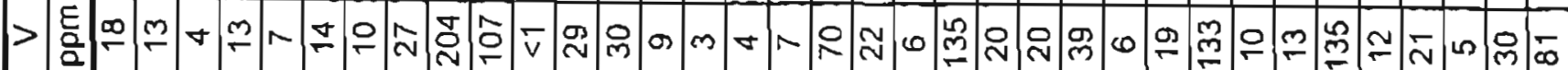

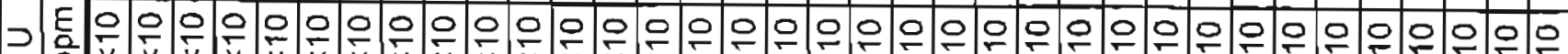

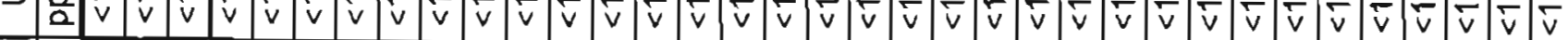

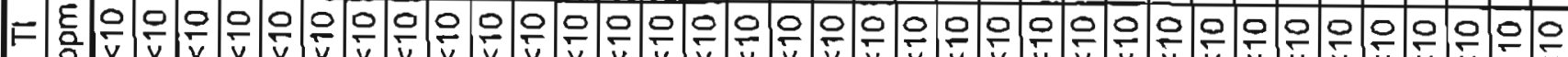

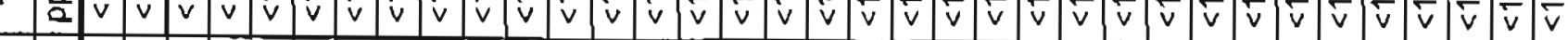
¡ $\approx$ \&

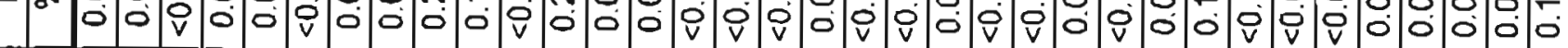
ī

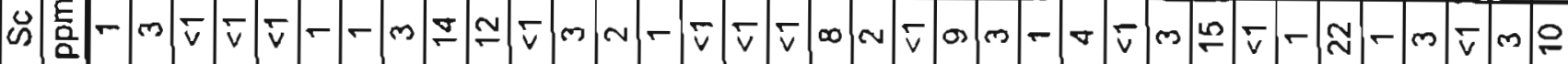

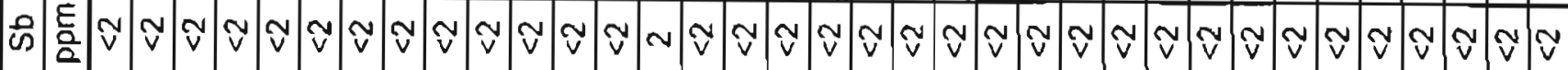
« ó

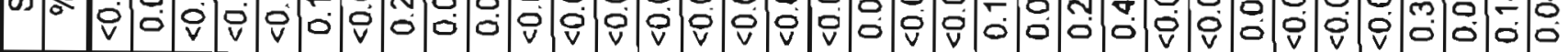
음 $\widetilde{a} \stackrel{0}{a}$

全

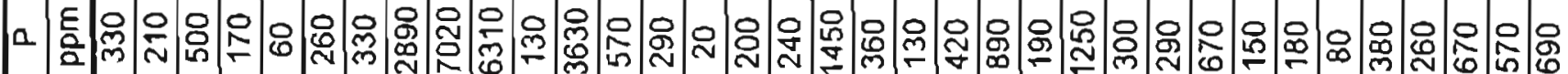

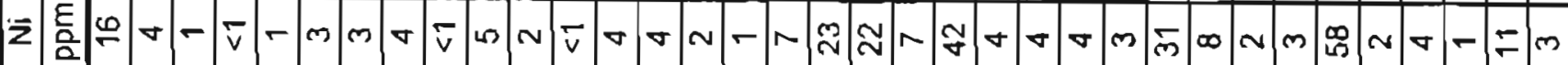
I

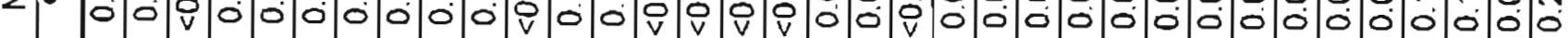

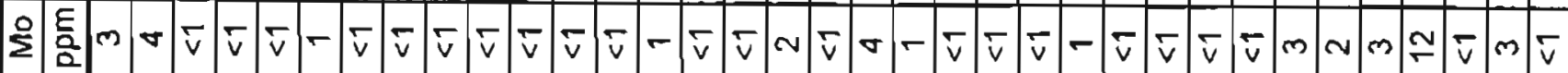

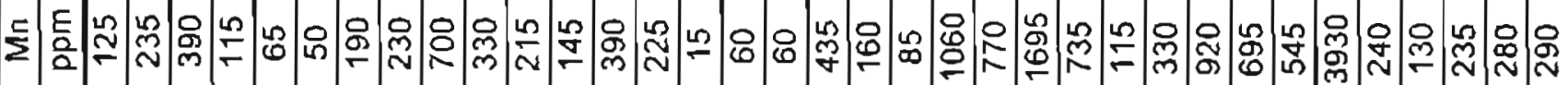

๖ำ

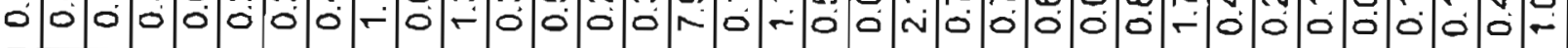

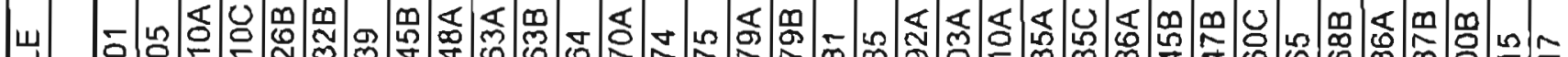

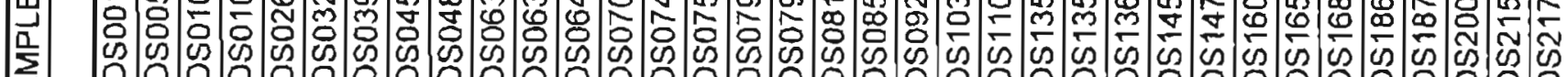

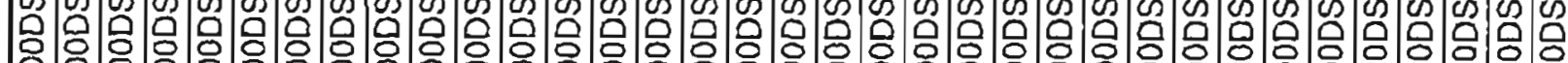
ผ 
Table 2. Concentration of trace elements in rock samples collected in the Eagle quadrangle. Note: --- = not analyzed.

\begin{tabular}{|c|c|c|c|c|c|c|c|c|c|c|c|c|c|c|c|c|c|c|c|}
\hline SAMPLE & $\mathrm{Mg}$ & $\mathrm{Mn}$ & Mo & $\mathrm{Na}$ & $\mathrm{Ni}$ & $P$ & $\mathrm{~Pb}$ & $P_{t}$ & $P d$ & $\mathrm{~S}$ & $\mathrm{Sb}$ & $\mathrm{Sc}$ & $\mathrm{St}$ & $\mathrm{Ti}$ & Ti & U' & V & W & $\overline{Z n}$ \\
\hline & $\%$ & $\mathrm{ppm}$ & $\mathrm{ppm}$ & $\%$ & ppm & ppm & ppm & $p \rho b$ & ppb & $\%$ & $\mathrm{ppm}$ & ppm & ppm & $\%$ & ppm & ppm & $\mathrm{ppm}$ & $\mathrm{ppm}$ & $\mathrm{ppm}$ \\
\hline $00 J G 042 C-6 / 30$ & 0.13 & 140 & $<1$ & 0.07 & 2 & 90 & 2 & $\overline{-.-}$ & - & 0.01 & $<2$ & $<1$ & 23 & 0.01 & $<10$ & $<10$ & 14 & रा0 & 14 \\
\hline $00 J G 050 A-6 / \overline{4}$ & 0.15 & $20^{-}$ & $<1$ & 0.11 & 1 & 80 & 2 & - & - & $0 . \overline{09}$ & $<2$ & $<1$ & 54 & $<0.01$ & $<10$ & $<10$ & 1 & $<10$ & $\overline{6}$ \\
\hline 00JG050日-6/14 & 0.79 & 515 & $<1$ & 0.06 & 2 & 910 & 6 & $<5$ & $<2$ & 0.07 & $<2$ & 2 & 65 & 0.15 & $<10$ & $<10$ & 53 & $<10$ & 68 \\
\hline D0JG063-6/16 & 0.22 & 275 & $<1$ & 0.06 & 1 & 610 & $\overline{4}$ & $\overline{-}$ & $\cdots$ & $<0 . \overline{01}$ & $<2$ & 1 & 95 & 0.09 & $<10$ & $<10$ & 14 & $<10$ & 12 \\
\hline $00 \mathrm{JG} 063 \mathrm{X}-7 / 1$ & $\overline{1.81}$ & 1365 & 1 & 0.05 & $<1$ & 1220 & $<2$ & - & - & 0.11 & $<2$ & 9 & 96 & 0.14 & $<10$ & $<10$ & 162 & $<10$ & $\overline{94}$ \\
\hline $00 \mathrm{JG067-7/2}$ & 1.27 & 890 & 1 & 0.04 & 3 & $15 \overline{40}$ & 8 & $\cdots$ & $-\infty$ & 0.08 & $<2$ & 4 & 77 & 0.10 & $<10$ & $<10$ & 45 & $<10$ & 118 \\
\hline $00 \mathrm{JG072-6/19}$ & 1.09 & 355 & 1 & 0.06 & 2 & 260 & 6 & $\cdots$ & $m$ & 0.14 & $<2$ & 6 & 17 & 0.10 & $<10$ & $<10$ & 24 & $\overline{<10}$ & $\overline{54}$ \\
\hline $00 \mathrm{JG} 073 \mathrm{~A}-7 / 2$ & 0.56 & 490 & 2 & $0.0 \overline{3}$ & 13 & 570 & 6 & --- & $\ldots-$ & $0 . \overline{04}$ & $<\overline{2}$ & 1 & 12 & 0.05 & $<10$ & $<10$ & 36 & $<10$ & 38 \\
\hline $00 \mathrm{JG077-6/19}$ & 0.93 & $370^{-}$ & $<1$ & $0 . \overline{8}$ & 12 & 370 & $<2$ & - & --- & 0.38 & $<2$ & 13 & 42 & 0.17 & $<10$ & $<10$ & 144 & $<10$ & 22 \\
\hline 00 JG082-6/19 & 0.81 & $180^{-}$ & 2 & $0.1 \overline{0}$ & 29 & 440 & 16 & $=$ & - & $\overline{0.44}$ & $<2$ & 7 & 73 & 0.21 & $<10$ & $<10$ & 68 & $<10$ & 108 \\
\hline 00JG084-6/19 & 0.49 & 420 & $<1$ & 0.01 & 3 & 80 & $<2$ & - & $-\infty$ & 0.03 & $<2$ & 1 & 233 & 0.04 & $<10$ & $<10$ & 14 & $<10$ & 24 \\
\hline 00JG089B-7/4 & 0.06 & 55 & $<1$ & 0.02 & 1 & 80 & 2 & - & $\cdots$ & $<0.01$ & $<2$ & 1 & 10 & 0.03 & $<10$ & $<10$ & 6 & $<10$ & 10 \\
\hline 00.JG93B-7/9 & 0.05 & 60 & 1 & 0.06 & 1 & 30 & 4 & -- & $\cdots$ & $<0.01$ & $<2$ & $<1$ & 19 & $<0.01$ & $<10$ & $<10$ & 4 & $<70$ & 2 \\
\hline $00 \bar{J} 698-7 / 9$ & 1.71 & 445 & 1 & 0.07 & 22 & 1460 & $<2$ & -- & - & $0 . \overline{59}$ & $<2$ & 11 & 87 & 0.06 & $<10$ & $<10$ & 125 & $<10$ & 68 \\
\hline O0JG136-6/23 & 0.91 & 600 & $<1$ & $0.0 \overline{9}$ & 3 & 1070 & 4 & - & - & 0.06 & $<2$ & 3 & 130 & 0.12 & $<10$ & $<10$ & 41 & $<10$ & 80 \\
\hline $00 J G 137-6 / 23$ & 0.75 & 480 & $<1$ & 0.06 & 3 & 890 & $<2$ & --- & -- & 0.06 & $<2$ & 2 & 78 & 0.08 & $<10$ & $<10$ & 38 & $<10$ & 72 \\
\hline $00 J G 138-6 / 23$ & 0.56 & 315 & $<1$ & 0.04 & 2 & 840 & $<2$ & $--\infty$ & $-\cdots$ & 0.05 & $<2$ & 1 & 101 & 0.07 & $<10$ & $<10$ & 21 & $<10$ & 54 \\
\hline $00 \mathrm{JG} 139 \cdot 6 / 23$ & 0.57 & 255 & $\angle 1$ & 0.04 & 1 & 790 & $<2$ & -- & $\cdots$ & 0.06 & $<2$ & $<1$ & 90 & 0.09 & $<10$ & $<10$ & 14 & $<10$ & 60 \\
\hline $00.5 G 140-6 / 23$ & 1.62 & 315 & $<1$ & 0.11 & 20 & 100 & 2 & - & - & 0.09 & $<2$ & 7 & 11 & 0.11 & $<10$ & $<10$ & 89 & $<10$ & 86 \\
\hline $00 J G 156-6 / 25$ & 0.17 & 50 & 3 & $0.0 \overline{7}$ & 5 & 100 & 2 & $=$ & - & $0 . \overline{08}$ & $<\overline{2}$ & $<1$ & 27 & 0.01 & $<10$ & $<10$ & 7 & $\angle 10$ & 26 \\
\hline 00JGSKOID\#1-6 & 0.03 & 315 & 1 & $<0.01$ & 5 & 110 & $<2$ & - & --- & 0.01 & $<2$ & $<1$ & 236 & 0.01 & $<10$ & $<10$ & 1 & $<10$ & $<2$ \\
\hline 00JGSKOID\#2-6/15 & 0.06 & 140 & 4 & 0.04 & 5 & 500 & $<2$ & -- & - & 0.01 & $<2$ & $<1$ & 78 & 0.13 & $<10$ & $<10$ & 10 & $<10$ & 2 \\
\hline OOMBW $11 \mathrm{~A}$ & 0.13 & 40 & $<1$ & 0.11 & $<1$ & 280 & 4 & -- & - & 0.46 & $<2$ & 8 & 66 & 0.05 & $<10$ & $<10$ & 16 & $<10$ & 8 \\
\hline OOMBW38A & 0.01 & 55 & $<i$ & $<0.01$ & 3 & 10 & $<2$ & - & - & $<0.01$ & $<2$ & $<1$ & 3 & $<0.01$ & $<10$ & $<10$ & 3 & $<10$ & 2 \\
\hline 00MBW73 & 0.47 & 685 & $<1$ & 0.09 & 4 & 400 & $<2$ & -- & $\cdots$ & $<0.01$ & $<2$ & 5 & 17 & 0.13 & $<10$ & $<10$ & 22 & $<10$ & 40 \\
\hline 00MBW94B & 0.74 & 415 & $<1$ & 0.10 & 11 & 330 & 8 & - & - & $0 . \overline{35}$ & $<2$ & 5 & 10 & 0.17 & $<10$ & $<10$ & 47 & $<10$ & 226 \\
\hline $00 \mathrm{MBW} 101 \mathrm{~A}$ & 0.14 & 200 & 1 & 0.08 & 1 & 1020 & 2 & $\cdots$ & -- & $<0.01$ & $<2$ & 1 & 538 & 0.08 & $<10$ & $<10$ & 27 & $<10$ & 16 \\
\hline $00 \mathrm{MBW} 101 \mathrm{C}$ & 0.01 & 505 & $<1$ & $<0 . \overline{01}$ & 2 & 110 & $<2$ & $\because$ & $=$ & $<0.01$ & $<2$ & $<1$ & 4 & $<0.01$ & $<10$ & $<10$ & 9 & $<10$ & 14 \\
\hline 00MBW104 & 0.08 & 875 & 1 & $<0.01$ & 3 & 760 & 22 & - & - & $<0.01$ & $<2$ & 2 & 37 & $<0.01$ & $<10$ & $<10$ & 21 & $<10$ & 46 \\
\hline OOMBW111 & 0.42 & 280 & 1 & 0.07 & 6 & 1180 & 2 & - & -- & 0.05 & $<2$ & 3 & 23 & $<0.01$ & $<10$ & $<10$ & 34 & $<10$ & 18 \\
\hline OOMBW112 & 0.03 & 1860 & $<1$ & $<0.01$ & 16 & 560 & 2 & - & $\ldots$ & $<0.01$ & $<2$ & 1 & 13 & $<0.01$ & $<10$ & $<10$ & 34 & $<10$ & 54 \\
\hline OOMBW130 & 0.04 & 50 & $\leq 1$ & $<0.01$ & 14 & 100 & $<2$ & - & - & 0.04 & $<2$ & 1 & 15 & $<0.01$ & $<10$ & $<10$ & 11 & $<10$ & 14 \\
\hline$\overline{00 M B W 142 B}$ & 0.06 & 115 & 1 & 0.04 & 5 & 270 & 22 & $=$ & - & $0 . \overline{05}$ & $<2$ & 2 & 14 & $<0.01$ & $<10$ & $<10$ & 25 & $<10$ & 20 \\
\hline OOMBW142C & 0.06 & 30 & $<1$ & 0.01 & 4 & 100 & 12 & -- & -- & $<0 . \overline{01}$ & $<2$ & $<1$ & 11 & $<0.01$ & $<10$ & $<10$ & 2 & $<10$ & 18 \\
\hline OOMBW180 & 0.56 & 180 & 1 & 0.03 & 17 & 130 & $<2$ & $\cdots$ & -- & $<0 . \overline{01}$ & $<2$ & $<1$ & 8 & 0.03 & $<10$ & $<10$ & 3 & $<10$ & 6 \\
\hline
\end{tabular}


Table 2. Concentration of trace elenents in rock samples collected in the Eagle quadrangle. Note; --- = not analyzed.

\begin{tabular}{|c|c|c|c|c|c|c|c|c|c|c|c|c|c|c|c|c|c|c|c|}
\hline SAMPLE & $\mathrm{Mg}$ & $\mathrm{Mn}$ & Mo & $\mathrm{Na}$ & $\mathrm{Ni}$ & $P$ & $\mathrm{~Pb}$ & Pt & $\mathrm{Pd}$ & $S$ & $S b$ & $\mathrm{Sc}$ & $\mathrm{Sr}$ & Ti & $T !$ & $\mathrm{U}$ & V & W & $\mathrm{Zn}$ \\
\hline & $\%$ & $\mathrm{ppm}$ & ppm & $\%$ & $\mathrm{ppm}$ & $\mathrm{ppm}$ & $\mathrm{ppm}$ & $\mathrm{ppb}$ & $\mathrm{ppb}$ & $\%$ & $\overline{\mathrm{ppm}}$ & ppm & ppm & $\%$ & ppm & ррпा & ppm & ppm & ppm \\
\hline $00 \mathrm{MBW} 187 \mathrm{~B}$ & 0.01 & 60 & $<1$ & 0.06 & 1 & 30 & 10 & $\overline{--}$ & $\cdots$ & $<0.01$ & $<2$ & $<1$ & 20 & $<0.01$ & $<10$ & $<10$ & $<1$ & $<10$ & 14 \\
\hline OOMBW190 & 0.31 & $40 \overline{0}$ & 7 & 0.04 & 5 & $3 \overline{7} 0$ & 6 & - & $\cdots$ & 0.07 & $<2$ & $<1$ & 69 & 0.01 & $<10$ & $<10$ & 7 & 120 & 70 \\
\hline OOMBW191 & 0.28 & 215 & 45 & 0.23 & 13 & 690 & 36 & - & $\cdots$ & 0.18 & $<2$ & 3 & 139 & 0.09 & $<10$ & $<10$ & 35 & $<\overline{10}$ & 212 \\
\hline OOMBW192 & 0.15 & 175 & 26 & 0.33 & 1 & 250 & 276 & - & -- & 0.09 & $<2$ & $<1$ & 203 & 0.02 & $<10$ & $<10$ & 9 & $<10$ & 550 \\
\hline OOMBW193 & 0.01 & 105 & 5 & 0.06 & 1 & 30 & 6 & - & $=$ & $<0.01$ & $<2$ & $<1$ & 23 & $<0.01$ & $<10$ & $<10$ & 1 & $<10$ & 12 \\
\hline COMBW200 & 1.45 & 315 & 1 & 0.04 & 5 & 900 & 4 & $\cdots$ & $\cdots$ & $<0.01$ & $<2$ & 5 & 96 & 0.05 & $<10$ & $<10$ & 42 & $<\overline{10}$ & 40 \\
\hline OOMBW215B & 0.22 & 140 & 4 & 0.04 & 4 & 210 & $\overline{6}$ & $=$ & - & 0.01 & $<2$ & 2 & 20 & 0.04 & $<10$ & $<10$ & 15 & $<10$ & 504 \\
\hline OOMBW 217 & 0.12 & 1140 & 1 & 0.06 & 6 & 230 & 6 & - & $\ldots$ & $<0.01$ & $<2$ & 1 & 26 & 0.01 & $<10$ & $<10$ & 18 & 10 & 850 \\
\hline $00 \mathrm{MBN} 224$ & 0.08 & 60 & $<1$ & 0.08 & 1 & 140 & 2 & - & $m$ & 0.04 & $<2$ & 3 & 42 & 0.02 & $<10$ & $<10$ & 5 & $<10$ & 20 \\
\hline 0OMBW230 & 0.13 & 100 & 4 & 0.02 & 5 & 130 & 2 & - & - & 0.03 & $<2$ & $<1$ & 16 & $<0.01$ & $<10$ & $<10$ & 6 & $<10$ & 24 \\
\hline $00 \mathrm{MBW} 250$ & 0.33 & 120 & 1 & 0.05 & 2 & 60 & 4 & - & - & $<0.01$ & $<2$ & 1 & 7 & 0.03 & $<10$ & $<10$ & 3 & $<10$ & 26 \\
\hline OONBW25 & 0.01 & 15 & $<1$ & 0.01 & 5 & 20 & 2 & - & $\cdots$ & $<0.01$ & $<2$ & $<1$ & 2 & $<0.01$ & $<10$ & $<10$ & 1 & $<10$ & $<2$ \\
\hline DOMBW264 & 0.17 & 990 & $<1$ & $<0.01$ & 11 & 120 & $<2$ & $\cdots$ & - & $<0.01$ & $<2$ & 3 & 3 & $<0.01$ & $<10$ & $<10$ & 16 & $<10$ & 14 \\
\hline 00MBW269 & 0.32 & 145 & 1 & $<0.01$ & 12 & 60 & 2 & $\infty$ & - & $<0.01$ & $<2$ & 1 & 1 & 0.01 & $<10$ & $<10$ & 32 & $<10$ & 18 \\
\hline $00 \mathrm{MBW} 280$ & 2.34 & 365 & 3 & 0.01 & 4 & 680 & $<2$ & - & - & 0.24 & $<2$ & 14 & 6 & 0.15 & $<10$ & $<10$ & 167 & $<10$ & 38 \\
\hline 00MBW281 & 1.42 & 390 & 4 & 0.05 & 8 & 450 & 26 & -- & $\cdots$ & 0.08 & $<2$ & 7 & 13 & 0.06 & $<10$ & $<10$ & 37 & $<10$ & 134 \\
\hline 00MBW306 & 0.46 & 285 & 20 & 0.07 & 11 & 500 & 10 & -- & $-\cdots$ & 0.24 & $<2$ & 4 & 58 & $<001$ & $<10$ & $<10$ & 45 & $<10$ & 62 \\
\hline 00MBW307A & 1.02 & 505 & 3 & 0.07 & 3 & 1780 & $<2$ & $\cdots$ & -- & $<0.01$ & $<2$ & 8 & 61 & 0.10 & $<10$ & $<10$ & 52 & $<10$ & 48 \\
\hline 00MBW308B & 0.88 & $4 \overline{30}$ & 5 & 0.01 & 6 & 280 & 48 & $\cdots$ & $\cdots$ & 0.01 & $<2$ & 5 & 5 & 0.01 & $<10^{\circ}$ & $<10$ & 84 & $<10$ & 42 \\
\hline OOMBW310B & 0.05 & 55 & 1 & $<0.01$ & 4 & 80 & 26 & $\cdots$ & $-\cdots$ & $<0.01$ & $<2$ & $<1$ & 3 & $<0.01$ & $<10$ & $<10$ & 6 & $<10$ & 22 \\
\hline OOMBW311B & 0.01 & 35 & 1 & $<0.01$ & 4 & 30 & $<2$ & -- & -- & $<0.01$ & $<2$ & $<1$ & 1 & $<0.01$ & $<10$ & $<10$ & 5 & $<10$ & 4 \\
\hline O0MBW321 & 0.72 & 350 & $<1$ & 0.05 & 4 & 210 & $<2$ & - & $\cdots$ & $<0.01$ & $<2$ & 2 & 4 & $<0.01$ & $<10$ & $<10$ & 18 & $<10$ & 54 \\
\hline OOMBW343 & 0.01 & 15 & 3 & 0.11 & 1 & 80 & $<2$ & - & - & 0.10 & $<2$ & $<1$ & 38 & $<0.01$ & $<10$ & $<10$ & 3 & $<10$ & $<2$ \\
\hline OOMBW351 & $0.77^{-}$ & 265 & $<1$ & 0.11 & 6 & 810 & $<2$ & - & --- & $<0.01$ & $<2$ & 5 & 24 & 0.07 & $<10$ & $<10$ & 35 & $<10$ & 32 \\
\hline 00MBW354 & 0.06 & 40 & 2 & 0.09 & 4 & 260 & $<2$ & $\cdots$ & $\rightarrow$ & $<0.01$ & $<2$ & $<1$ & 38 & $<0.01$ & $<10$ & $<10$ & 6 & $<10$ & 18 \\
\hline $00 \mathrm{MBW} 355 \mathrm{C}$ & 0.05 & 25 & 4 & $\angle 0.01$ & 7 & 380 & $<2$ & - & - & 0.22 & $<2$ & $\leq 1$ & 16 & $<0.01$ & $<10$ & $<10$ & 6 & $<10$ & 6 \\
\hline $00 \mathrm{MBW} 356 \mathrm{~A}$ & 0.03 & 200 & 1 & 0.06 & 5 & 60 & 2 & $\cdots$ & - & 0.10 & 2 & $<1$ & 25 & $<0.01$ & $<10$ & $<10$ & 1 & $<10$ & 16 \\
\hline OOMBW356日 & 0.13 & 105 & 1 & 0.10 & 4 & 110 & 6 & $\cdots$ & - & 0.08 & $<2$ & $<1$ & 51 & $<0.01$ & $<10$ & $<10$ & 4 & $<10$ & 30 \\
\hline OOMBW384 & 0.19 & $200^{\circ}$ & 2 & 0.09 & 3 & 90 & $<2$ & - & - & 0.34 & $<2$ & $<1$ & 39 & $<0.01$ & $<10$ & $<10$ & 7 & $<10$ & 22 \\
\hline OOMBW443 & 0.91 & 1005 & 3 & 0.02 & 18 & 500 & 8 & - & - & $<0.01$ & $<2$ & 4 & 66 & $<0.01$ & $<10$ & $<10$ & 24 & $<10$ & 64 \\
\hline OOMBW460B & 1.38 & 680 & 2 & 0.08 & 4 & 880 & $<2$ & - & -- & 0.05 & $<2$ & 8 & 13 & 0.17 & $<10$ & $<10$ & 81 & $<10$ & 86 \\
\hline $00 \mathrm{MBW} 461 \mathrm{~A}$ & 1.65 & 645 & 1 & 0.05 & $<1$ & 620 & 2 & - & -- & $<0.01$ & $<2$ & 5 & 28 & 0.07 & $<10$ & $<10$ & 52 & $<10$ & 152 \\
\hline $00 \mathrm{MBN} 469$ & 0.22 & 165 & 3 & 0.08 & 4 & 220 & 2 & - & - & 0.01 & $<2$ & 2 & 48 & 0.06 & $<10$ & $<10$ & 21 & $<10$ & 52 \\
\hline OOMBW513 & 0.04 & 20 & 2 & 0.09 & 2 & 30 & $<2$ & - & - & $<0.01$ & $<2$ & $<1$ & 19 & $<0.01$ & $<10$ & $<10$ & 3 & $<10$ & 4 \\
\hline OOMBW523 & 0.09 & 35 & 5 & 0.01 & 5 & 80 & $<2$ & - & - & 0.01 & $<2$ & $<1$ & $<1$ & $<0.01$ & $<10$ & $<10$ & 3 & $<10$ & 2 \\
\hline
\end{tabular}


Table 2. Concentration of trace elements in rock samples collected in the Eagle quadrangle. Note: --- = not analyzed.

\begin{tabular}{|c|c|c|c|c|c|c|c|c|c|c|c|c|c|c|c|c|c|c|c|}
\hline SAMPLE & $\mathrm{Mg}$ & $\mathrm{Mn}$ & Mo & $\mathrm{Na}$ & $\mathrm{Ni}$ & $P$ & $\mathrm{~Pb}$ & $\mathrm{Pt}$ & Pd & $\mathrm{S}$ & $\mathrm{Sb}$ & Sc & $\mathrm{Sr}$ & $\overline{\mathrm{Ti}}$ & TI & $\bar{U}$ & $\bar{v}$ & $\bar{W}$ & $2 n$ \\
\hline & $\%$ & ppm & ppm & $\%$ & ppm & ppm & $\mathrm{ppm}$ & $p p b$ & ppb & $\%$ & ppm & $\mathrm{ppm}$ & $\mathrm{ppm}$ & $\%$ & ppm & ppm & ppm & ppm & $\mathrm{ppm}$ \\
\hline $00 \mathrm{MBW} 545$ & 0.18 & 195 & $<1$ & $\overline{0.09}$ & 1 & 550 & 4 & - & - & 0.14 & $<2$ & 3 & 24 & 0.08 & $<10$ & $<10$ & 20 & $<10$ & 20 \\
\hline OOMBW546C & $0 . \dot{0} 2$ & $45^{-}$ & $<1$ & 0.05 & 3 & 80 & 4 & - & -- & 0.20 & $<2$ & $<1$ & 20 & $<0.01$ & $<10$ & $<10$ & 1 & $<10$ & 2 \\
\hline O0MBW564 & 1.25 & 535 & 6 & 0.06 & 7 & 590 & $<2$ & - & - & 0.80 & $<2$ & 11 & 14 & 0.12 & $<10$ & $<10$ & 77 & $<10$ & 66 \\
\hline$\overline{00 M B W 568 A}$ & 1.65 & 940 & 1 & 0.05 & $\widetilde{4}$ & 1810 & 2 & - & - & 1.88 & $<2$ & 9 & 108 & $\overline{0.01}$ & $<10$ & $<10$ & $\overline{137}$ & $<10$ & 140 \\
\hline OOMBW568C & 0.98 & 580 & 3 & 0.03 & 10 & 840 & $<2$ & - & - & 0.73 & $<2$ & 10 & 33 & $\overline{0.08}$ & $<10$ & $<10$ & $\overline{106}$ & $<10$ & 90 \\
\hline $00 \mathrm{MBW} 570$ & 1.32 & 315 & 1 & 0.10 & 35 & $630^{\circ}$ & 2 & - & $\cdots$ & 0.12 & $<2$ & 6 & 39 & 0.10 & $<10$ & $<10$ & 57 & $<10$ & 28 \\
\hline 00MBW571 & 0.52 & $11 \overline{65}$ & $<1$ & 0.01 & 7 & 320 & $<2$ & $\ldots$ & - & 0.08 & $<2$ & 3 & 343 & 0.04 & $<10$ & $<10$ & 27 & $<10$ & 10 \\
\hline O0RN62 & 0.66 & 785 & 1 & 0.08 & 8 & 350 & 4 & -- & - & 0.03 & $<2$ & 10 & 16 & 0.20 & $<10$ & $<10$ & 39 & $<10$ & 86 \\
\hline$\overline{00 R N} \overline{96} \mathrm{C}$ & $0 . \overline{59}$ & 120 & 1 & 0.08 & 1 & $26 \overline{0}$ & 2 & - & $\cdots$ & 0.69 & $<2$ & 15 & 61 & 0.05 & $<10$ & $<10$ & 67 & $<10$ & 28 \\
\hline DORN102 & $<0.01$ & 95 & 1 & $<0.01$ & 8 & 150 & 6 & - & $=$ & $<0.01$ & 76 & 1 & 4 & $<0.01$ & $<10$ & $<10$ & 7 & $<10$ & 32 \\
\hline OORN109 & 0.04 & 165 & $<1$ & 0.21 & 3 & 410 & 28 & - & $\cdots$ & 0.03 & $<2$ & $<1$ & 79 & 0.06 & $<10$ & $<10$ & 6 & $<10$ & 40 \\
\hline$\overline{\text { OORN147B }}$ & 2.53 & 250 & $<1$ & $<0.01$ & 1 & 340 & $<2$ & - & -- & $<0.01$ & $<2$ & $<1$ & 80 & $<0.01$ & $<10$ & $<10$ & 7 & $<10$ & 14 \\
\hline OORN147C & 0.99 & 525 & $<1$ & 0.17 & 6 & 1920 & $<2$ & - & $\cdots$ & 0.30 & $<2$ & 10 & 80 & 0.16 & $<10$ & $<10$ & 85 & $<10$ & 64 \\
\hline OORN147D & 0.16 & 135 & $<1$ & 0.08 & 1 & 280 & 6 & $\cdots$ & - & 0.10 & $<2$ & 1 & 55 & 0.04 & $<10$ & $<10$ & 11 & $<10$ & 16 \\
\hline$\overline{O O R N 154}$ & $0 . \overline{50}$ & 155 & $<1$ & $<0.01$ & 3 & 30 & $<2$ & $\cdots$ & -- & $<0.01$ & $<2$ & $<1$ & 341 & $<0.01$ & $<10$ & $<10$ & 5 & $<10$ & $<2$ \\
\hline$\overline{00 R N 165}$ & 0.64 & 385 & $<1$ & 0.10 & 3 & 910 & $<2$ & $\cdots$ & $\cdots$ & 0.19 & $<2$ & 4 & 59 & 0.11 & $<10$ & $<10$ & 44 & $<10$ & 56 \\
\hline OORN175A & 0.38 & 420 & $<1$ & 0.04 & 4 & 1010 & $<2$ & 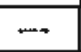 & --- & $<0.01$ & $<2$ & 4 & 83 & $\overline{0.07}$ & $<10$ & $<10$ & 31 & $<10$ & 26 \\
\hline OORN192A & 0.20 & 115 & $<1$ & 0.07 & 1 & 1120 & 4 & $-\infty$ & $\cdots$ & 0.01 & $<2$ & 1 & 307 & 0.07 & $<1 \overline{0}$ & $<10$ & 16 & $<10$ & 10 \\
\hline O0RN192B & 2.33 & 260 & $<1$ & 0.01 & $<1$ & 630 & 4 & - & -- & $<0.01$ & $<2$ & $<1$ & 102 & 0.03 & $<10$ & $<10$ & 8 & $<10$ & 16 \\
\hline OORN244A & 0.29 & 360 & $<1$ & 0.07 & 7 & 880 & 6 & -- & $\cdots$ & $<0.01$ & $<2$ & 3 & 57 & 0.09 & $<10$ & $<10$ & 24 & $<10$ & 34 \\
\hline O0RN252A & $1 . \overline{90}$ & 600 & 41 & 0.08 & 28 & 1210 & $<2$ & $\cdots$ & - & 0.53 & 2 & 17 & 121 & $\overline{0.13}$ & $<10$ & $<10$ & 121 & $<10$ & 60 \\
\hline OORN254 & $0 . \overline{01}$ & $35^{-}$ & $<1$ & $\overline{0.07}$ & 1 & 220 & 2 & -- & - & $<0,01$ & $<2$ & $<1$ & 8 & $<0.01$ & $<10$ & $<10$ & 1 & $<10$ & 2 \\
\hline 00RN255C & 0.09 & 65 & $<1$ & 0.01 & 3 & 170 & $<2$ & -- & --- & $<0.01$ & $<2$ & $<1$ & 3 & 0.02 & $<10$ & $<10$ & 11 & 510 & 10 \\
\hline OORN256C & 0.53 & 240 & $<1$ & 0.02 & 20 & 2170 & $<2$ & --- & - & $<0.01$ & $<2$ & 1 & 21 & 0.16 & $<10$ & $<10$ & 25 & $<10$ & 22 \\
\hline CORN322A & 0.33 & 320 & $<1$ & 0.01 & 6 & 700 & 4 & $\cdots$ & - & 0.03 & $<2$ & 1 & 98 & 0.05 & $<10$ & $<10$ & 16 & $<10$ & 30 \\
\hline 00RN325 & $0 . \overline{44}$ & 350 & $<1$ & 0.17 & $<1$ & 4480 & $<2$ & $<5$ & $<2$ & 0.62 & $<2$ & 5 & 61 & 0.36 & $<10$ & $<10$ & 300 & $<10$ & 54 \\
\hline OORN330A & 0.03 & 295 & 1 & $<0.01$ & 6 & 300 & 2 & - & -- & $<0.01$ & $<2$ & 1 & 12 & $<0.01$ & $<10$ & $<10$ & 11 & $<10$ & 10 \\
\hline O0RN331 & 2.95 & 555 & $<1$ & 0.01 & 268 & 60 & $<2$ & - & - & 1.13 & $<2$ & 30 & 92 & 0.01 & $<10$ & $<10$ & 106 & $<10$ & 26 \\
\hline 00RN332 & 1.75 & 415 & $<1$ & 0.03 & 333 & 40 & 2 & $\cdots$ & - & 1.41 & $<2$ & 16 & 145 & 0.02 & $\angle 10$ & $<10$ & 57 & $<10$ & 20 \\
\hline DORN333 & 0.94 & 350 & $<1$ & 0.05 & 36 & 60 & $<2$ & $\cdots$ & $\cdots$ & 0.07 & $<2$ & 12 & 21 & 0.06 & $<10$ & $<10$ & 47 & $<10$ & 16 \\
\hline 00RN334 & $<0.01$ & 25 & 1 & 0.04 & 1 & 30 & 34 & -- & - & $<0.01$ & $<2$ & $<1$ & 1 & $<0.01$ & $<10$ & $<10$ & 1 & $<10$ & 16 \\
\hline 00RN336 & $<0.01$ & 15 & 4 & 0.05 & 1 & 90 & 28 & - & - & 0.12 & $<2$ & $<1$ & 4 & $\overline{<0.01}$ & $<10$ & $\angle 10$ & 1 & $<10$ & 16 \\
\hline 00RN337B & 0.02 & 275 & $<1$ & $<0.01$ & 3 & 10 & $<2$ & - & - & $<0.01$ & $<2$ & $<1$ & 9 & $<0.01$ & $<10$ & $<10$ & 1 & $<10$ & 2 \\
\hline 00RN338A & $0 . \overline{0} \overline{1}$ & 25 & $<1$ & 0.03 & 1 & 280 & 2 & - & -- & $<0.01$ & $<2$ & 2 & 6 & 0.01 & 10 & $<10$ & 5 & $<10$ & 44 \\
\hline OORN480A & 0.15 & 270 & 2 & 0.13 & 7 & 100 & $<2$ & - & - & 0.25 & $<2$ & 1 & 98 & $<0.01$ & $<10$ & $<10$ & 8 & $<10$ & 26 \\
\hline
\end{tabular}


Table 2. Concentration of trace elements in rock samples collected in the Eagle quadrangle. Note: ..- = not analyzed.

\begin{tabular}{|c|c|c|c|c|c|c|c|c|c|c|c|c|c|c|c|c|c|c|c|}
\hline SAMPLE & $\mathrm{Mg}$ & Мл & Mo & $\mathrm{Na}$ & $\mathrm{Ni}$ & $\mathrm{P}$ & $\mathrm{Pb}$ & $\mathrm{Pt}$ & Pd & $\$$ & $S b$ & $\mathrm{Sc}$ & $\mathrm{Sr}$ & $\mathrm{Ti}$ & $\mathrm{TI}$ & $\mathrm{U}$ & $\mathrm{V}$ & $W$ & $\mathrm{Zn}$ \\
\hline & $\%$ & $\mathrm{ppm}$ & ppm & $\%$ & $\mathrm{ppm}$ & $\mathrm{ppm}$ & $p \rho m$ & ppb & ppo & $\%$ & ppm & $\mathrm{ppm}$ & $\rho p m$ & $\%$ & ppm & $\mathrm{ppm}$ & ppm & ppm & ppm \\
\hline O0RN481A & 0.69 & 4790 & 4 & 0.01 & 126 & 1320 & $<2$ & $\cdots$ & - & 2.10 & $<2$ & 7 & 145 & $<0.01$ & $<10$ & $<10$ & 56 & $<10$ & 194 \\
\hline O0RN $481 \mathrm{~B}$ & 0.30 & 495 & 1 & $0 . \overline{01}$ & 17 & 990 & $<2$ & - & - & 0.76 & $<2$ & 1 & 24 & $<0.01$ & $<10$ & $<10$ & 22 & $<10$ & 30 \\
\hline 00RN493A & 1.78 & 465 & 3 & $<0.01$ & 46 & 980 & $<2$ & -- & $\cdots$ & $1 . \overline{46}$ & $<2$ & 7 & 34 & 0.21 & $<10$ & $<10$ & 87 & $<10$ & 82 \\
\hline O0RN515A & 0.04 & 20 & $<1$ & $0 . \overline{0}$ & 4 & 440 & 10 & 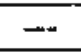 & $-\infty$ & $0 . \overline{77}$ & $<2$ & 3 & 12 & $<0.01$ & $<30$ & $<10$ & 8 & $<10$ & 12 \\
\hline OORN515B & $<0.01$ & 10 & 1 & 0.08 & 3 & 30 & $<2$ & $-m$ & $\ldots$ & $0 . \overline{40}$ & $<2$ & $<1$ & 70 & $<0.01$ & $<10$ & $<10$ & 1 & $<10$ & $<2$ \\
\hline$\overline{00 R N 515 \bar{C}}$ & 1.53 & 335 & 2 & $0 . \overline{19}$ & 59 & 890 & $<\overline{2}$ & $<5$ & $<2$ & $>5.00$ & $<2$ & 6 & 31 & 0.18 & $<10$ & $<10$ & $\overline{146}$ & 10 & $\overline{68}$ \\
\hline 00RN523A & 0.45 & 230 & 6 & 0.02 & 57 & 290 & $<2$ & $\cdots$ & - & 0.77 & $<2$ & 1 & 35 & $<0.01$ & $<10$ & $<10$ & 44 & $<10$ & 38 \\
\hline 00RN523B & 0.26 & 505 & 1 & $<0.01$ & 6 & 460 & $<2$ & 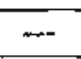 & $\cdots$ & 0.91 & $<2$ & $<1$ & 101 & $<0.01$ & $<10$ & $<10$ & 8 & $<10$ & 56 \\
\hline 00RN533B & 0.05 & 35 & $<1$ & 0.03 & 4 & 40 & 4 & $\cdots$ & - & 0.01 & $<2$ & 4 & 8 & $<0.01$ & $<10$ & $<10$ & 9 & $<10$ & 10 \\
\hline OORN560 & 0.01 & 190 & $<1$ & 0.06 & 8 & 50 & 2 & $\cdots$ & $m$ & $0 . \overline{0}$ & 2 & $\overline{1}$ & 5 & $<0.01$ & $<10$ & $<10$ & 9 & $<10$ & 20 \\
\hline OOWM133 & 1.49 & 455 & 1 & 0.07 & 1 & 340 & 2 & --- & 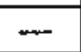 & 0.27 & $<2$ & 8 & 29 & 0.19 & $<10$ & $<10$ & 61 & $<10$ & 48 \\
\hline $00 \mathrm{WM} 144 \mathrm{C}$ & 2.02 & 265 & $<1$ & 0.04 & 3 & 670 & 2 & -- & 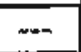 & 1.03 & $<2$ & 21 & 15 & 0.11 & $<10$ & $<10$ & 113 & $<10$ & 34 \\
\hline OOWM151 & 0.11 & 70 & 11 & 0.09 & 1 & 550 & 2 & -- & $\cdots$ & $0 . \overline{60}$ & $<2$ & 10 & 54 & 0.03 & $<10$ & $<10$ & 72 & $<10$ & 18 \\
\hline 00 WM206 & 5.12 & 80 & $<1$ & 0.01 & 2 & 100 & $<2$ & $-\cdots$ & --- & $<0.01$ & $<2$ & $<1$ & 234 & $<0.01$ & $<10$ & $<10$ & 3 & $<10$ & $<2$ \\
\hline O0WM210 & 3.27 & 625 & $<1$ & 0.06 & 145 & 4190 & $<2$ & $\because$ & - & $<0.01$ & $<2$ & 3 & 134 & 0.27 & $<10$ & $<10$ & 123 & $<10$ & 90 \\
\hline O0WM269B & 0.02 & 15 & 1 & $<0.01$ & 5 & 180 & 2 & 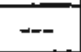 & - & 0.08 & $<2$ & $<1$ & 15 & $<0.01$ & $<10$ & $<10$ & 8 & $<10$ & $<2$ \\
\hline 00WM $369 \mathrm{~B}$ & 0.03 & 20 & 51 & 0.01 & 6 & 3080 & 1170 & -- & - & $0 . \overline{12}$ & 6 & $<1$ & 23 & $<0.01$ & $<10$ & $<10$ & 179 & $<10$ & 76 \\
\hline
\end{tabular}


Table 3. Location and description of rock samples collected in the Eagle and Tanacross quadrangles for major oxide, minor oxide, and trace element analyses.

\begin{tabular}{|c|c|c|c|}
\hline Sample \# & UTME & UTMN & Sample description \\
\hline 00DS009A & 465194 & 7113546 & White, slightly porphyritic, quastz feldspar white mica felsic dike. Quartz content $\sim 20 \%$. No foliation \\
\hline 0005020 & 453364 & 7119650 & $\begin{array}{l}\text { Biotite-rich amphibolite with } \sim 15 \% \text { biotite, }-30 \% \text { feidspar, and } \sim 60 \% \text { amphibole. Occasional light brown cm-wide } \\
\text { quartz veins parallel to foliation }\end{array}$ \\
\hline 00DS026B & 453099 & 7115691 & $\begin{array}{l}\text { Pegmatite veins up to } 5 \mathrm{~cm} \text { wide with K-feidspar-quartz-chlorite-epidote (chloritized biotile), sparse disseminated fine } \\
\text { grained magnetite }\end{array}$ \\
\hline $00 \mathrm{DS} 040$ & 451813 & 7118187 & Quartz-biotite-feldspar-white mica orthogneiss: Moderate iron oxide stain on foliation surfaces \\
\hline $0 \overline{00 S 042 \bar{A}}$ & 452637 & 7118265 & Leucocratic biotite quartz feldspar orthogneiss \\
\hline 0005053 & 457740 & 7116056 & $\begin{array}{l}\text { Augen gneiss with biolite and white to slightly pink augen eyes. Many quartz and quarz-feldspar veins parallel to } \\
\text { foliation in outcrop }\end{array}$ \\
\hline OODS058A & $4 \overline{4} \overline{85} 53$ & 7118506 & Biotite granodiorite: non-foliated (at best weakly foliaked) \\
\hline OODS058B & 458553 & 7118506 & Granitic pegmatile with red garnet phenocrysts $(2-3 \%)$ up to $2-3 \mathrm{~mm}$ in diameter \\
\hline $000 \mathrm{DS} 086$ & 431016 & $7 \overline{139769}$ & Black basalk with slight green cast to some plagioclase phenocrysts \\
\hline $00050 \overline{7}$ & 431020 & 7140160 & Black basalt with possible fine-grained biotste phenocrysts? \\
\hline 00DS100 & 454119 & 7098845 & Hornblende gabbro \\
\hline 00DS124 & 471642 & 7110590 & Biotite feldspar gneiss with weak chiorite alteration \\
\hline ODDS129 & 470940 & $7 \overline{109461}$ & Dark green to black amphibolite with hornblende phenocrysts up to $1.5 \mathrm{~cm}$ long \\
\hline 00DS135B & 469264 & 7115501 & Black and white metagabbro; sometimes with schlieren texture. Occurs as xenoliths or dikes within Napoleon pluton \\
\hline $00 D S 141$ & 469947 & 7114776 & Black and white hornblende bearing Napoleon (monzonite) pluton. Some minor alteration to chlorite \\
\hline $00 D S 160 \mathrm{~A}$ & 451855 & 7097520 & Black, partiafly sheared gabbro. Minor calcite veining \\
\hline OODS163 & 452524 & 7098854 & $\begin{array}{l}\text { Taylor Mountain Batholith hornblende-biotite granodiorite. Minor chlorite, epidote, and hematite on some joint and } \\
\text { fracture surfaces }\end{array}$ \\
\hline 0005168 & 455164 & 7101788 & $\begin{array}{l}\text { Gray, dacite porphyry with feldspar phenocrysts }(\sim 40 \% \text { of rock and up to } 1 \mathrm{~cm} \text { long by } 0.75 \mathrm{~cm} \text { across). Probable } \\
\text { dike }\end{array}$ \\
\hline $000 \$ 180$ & 474027 & 7110292 & Coarse-grained biotite amphibolite \\
\hline $000 S 185$ & 478467 & 7120018 & $\begin{array}{l}\text { Weakly foliated biolite-hornblende monzodiorite with } \sim 30-35 \% \text { biotile, } 10-15 \% \text { hornblende, }<5 \% \text { quartz, and several } \\
\text { small schlieren areas }\end{array}$ \\
\hline OODS215E & 463497 & 7119500 & Hornblende plagioclase gneiss (meta gabbro). Amphibole phenocrysts up to $1.5 \mathrm{~cm}$ long \\
\hline $00 D S 217$ & 463414 & 7119740 & Meta gabbro with large hornblende phenocrysts \\
\hline $00 \mathrm{~J} 6033 \sim 6 / 29$ & 479630 & 7107199 & Leucocratic orthogneiss \\
\hline 00JG047-6/30 & 478784 & $7 \uparrow 10027$ & Biotite orthogneiss \\
\hline $00 \mathrm{JG050 \textrm {B } - 6 / 1 4}$ & 455583 & 7122527 & Metagabbro with sulfides \\
\hline $00 J G 089-7 / 4$ & $47 \overline{3} 59$ & 7119161 & Orthogneiss \\
\hline $00 J G 094-6 / 20$ & 479275 & 7108835 & Orthogneiss \\
\hline $00 \sqrt{6097-6 / 20}$ & 478886 & 7107958 & Orthogneiss \\
\hline
\end{tabular}


Table 3. Location and description of rock samples collected in the Eagle and Tanacross quadrangles for major oxide, minor oxide, and trace element analyses.

\begin{tabular}{|c|c|c|c|}
\hline Sample \# & TTME & UTMN & Sample description \\
\hline $00 J G 125-6 / 22$ & 481652 & 7116329 & Clinopyroxenite dike \\
\hline $00.16153-6 / 25$ & 472028 & 7097155 & Muscovite-biotite (partially chloritized)-quartz-feldspar orthogneiss \\
\hline OOMBW $11 \mathrm{D}$ & 482538 & 7117989 & $\begin{array}{l}\text { Pale gray, finely banded quartzo-feldspathic gneiss with 2- to 3-mm-diameter equant feldspars floating in a finer- } \\
\text { grained matrix }\end{array}$ \\
\hline OOMBW18 & 480812 & 7118765 & $\begin{array}{l}\text { Pink and green, fine- to medium-grained, very foliated metaplutonic rock. Partially chloritized biotite (up to } 35-40 \% \text { ) } \\
\text { defines foliation }\end{array}$ \\
\hline OOMBW21 & 480946 & 7118569 & Pink and gray, medium grained, augen gneiss with relict K-feldspar crystals up to $2.5 \mathrm{~cm}$ long. \\
\hline OOMBW24A & 481137 & 7118313 & Very fine grained, black, finely foliated biotite amphibolite(?) \\
\hline 00MBW25B & 481193 & 7118286 & Slightly foliated, finely porphyritic biotite quartz diorite? with quart and feldspar phenocrysis up to $0.4 \mathrm{~cm}$ in diameter \\
\hline 00MBW26 & 481249 & 7118260 & Medium gray, fine grained, equigranular, quartz diorite(?) with $\sim 10 \%$ black, faintly-alligned biotite phenocrysts \\
\hline OOMBW43 & 465755 & 7122973 & Light gray, faintly foliated, fine-grained, hornblende-biotite quartz diorite or granodiorite \\
\hline 00MBW54 & 463896 & 7122548 & $\begin{array}{l}\text { Foliated phlogopite? clinopyroxenite? Appears porphyritic with phlogopitelclinopyroxene phenocrysts (up to } 1 \mathrm{~cm} \text { in } \\
\text { diameter) in a fine-grained, foliated, chlorite? matrix }\end{array}$ \\
\hline 00MBW55 & 463843 & 7122428 & Hornblende?/clinopyroxene? gabbro(?) \\
\hline OOMBN64 & 463586 & 7121383 & Dark green, finely foljated, biotite amphibolite \\
\hline $00 \mathrm{MBN93}$ & 467113 & 7117659 & White and tan, equigranular, barely foliated, hornblendeibiotite? monzogranite or granodiorite \\
\hline OOMBW97 & 467286 & 7119586 & White and black, medium grained, faintly foliated quartz diorite/monzodiorite? \\
\hline $00 \mathrm{MBW} 121$ & 472759 & 7115954 & Napoleon pluton: white and black, slightly foliated, equigranular, hornblende (15-20\%) quartz monzonite \\
\hline 00MBW126 & 468483 & 7114744 & $\begin{array}{l}\text { Napoleon pluton: white and green, medium grained, equigranular, hornblende (15-20\%) quartz monzonite. Slight to } \\
\text { moderate foliation }\end{array}$ \\
\hline $00 M B W 141$ & 466666 & 7112280 & White, slightly foliated aplite (rhyolite?) dike with no mafic minerals \\
\hline OOMBN143 & 466616 & 7111813 & White, fine grained, equigranular felsic intrusion \\
\hline OOMBW215A & 455994 & 7116863 & $\begin{array}{l}\text { K-feldspar porphyritic, fainlly foliated, biotite-rich }(\sim 50 \%) \text {, fine to medium grained, biotite quartz monzonite or } \\
\text { monzogranite }\end{array}$ \\
\hline 00MBW216 & 456073 & 7116870 & Tan, equigranular, foliated, pluton with $\sim 30 \%$ hornblende \\
\hline OOMBW243 & 464821 & 7109277 & Black, fine to medium grained, equigranular gabbro \\
\hline OOMBW313 & 437941 & 7146020 & Black, clinopyroxene-phyric basalt \\
\hline OOMBW318 & 436494 & 7145807 & Black, plagioclase?-phyric, intermediate? volcanic rock with aphanitic to almost glassy matrix \\
\hline 00MBW445B & 464404 & 7106993 & Green basalt/microgabbro \\
\hline 00MBW474 & 478225 & 7110487 & Light gray, fine to medium grained, planar foliated, biotite $(8 \%)$ quartz feldspar orthogneiss \\
\hline 00MBW507 & 485490 & 7118888 & Pink, fine grained, foliated, biotite ( $<5 \%)$ quartz feldspar orthogneiss \\
\hline 00MBW535 & 463440 & 7120077 & Dark green and white, medium grained, K-feldspar porphyritic, slightly foliated, biotite granodiorite/granite \\
\hline OOMBW570 & 462968 & 7123477 & Dark green, medium grained amphibolite with $\sim 3 \%$ disseminated iron sulfide \\
\hline $00 \mathrm{MBW} 575$ & 463318 & 7123594 & Dark green, medium grained, amphibole-plagioclase gneiss (metagabbro?) \\
\hline
\end{tabular}


Table 3. Location and description of rock samples collected in the Eagle and Tanacross quadrangles for major-oxide, minor oxide, and trace element analyses.

\begin{tabular}{|c|c|c|c|}
\hline Sample \# & UTWE & UTMN & Sample description \\
\hline O0RN34 & $4 \overline{777 \overline{9}}$ & 7062747 & Augen gneiss with huge K-feldspar phenocrysts. Tanacross quadrangle \\
\hline$\overline{00} \bar{R} \mathrm{~N} 48 \mathrm{C}$ & $4800 \overline{87}$ & $7 \overline{12426 \overline{6}}$ & Amphibolite \\
\hline 00RN51A & 480027 & 7123151 & Trondhjemitic orthogneiss? \\
\hline 00RN51B & 480027 & 7123151 & Augen gneiss \\
\hline DORN58 & 478550 & 7121500 & Magnetic amphibolite \\
\hline 00RN64 & 475507 & 7121368 & Fine grained amphibolite \\
\hline OORN73 & 469583 & 7122073 & $\begin{array}{l}\text { Uhler Pluton, boulder field of medium- to coarse-grained porphyritic hornblende biotite quartz monzodionite. Big } \\
\text { (white) K-feldspar in medium grained plagioclase-rich matrix with } \sim 15 \% \text { quartz }\end{array}$ \\
\hline $00 \mathrm{FN} 75$ & 469019 & 7122309 & Uhier pluton: slightly foliated, medium-grained, biotite conten $\sim 30 \%$; hornblende-biotite granodiorile \\
\hline 0ORN84 & 467179 & 7122412 & Uhler pluton: medium-grained, porphyritic biotite quartz monzodiorite \\
\hline OORN93 & $480 \overline{014}$ & $\overline{7119} 121$ & 'Great Dike': medium-grained, sub-equigranular, hornblende granodiorike dike \\
\hline DORN96A & 482502 & 7118013 & Biotite rich amphibofite \\
\hline OORN96B & 482502 & $71180+3$ & Orthogneiss, dikes?/sills in big pit; fine- to medium-grained biotite granodiorite/trondhjemite \\
\hline OORN99 & 483313 & 7117010 & $\begin{array}{l}\text { Orthogneiss near Steel Creek dome summit (elev. 3500'). Strongly foliated biolite tonalite. Coarse grained } \\
\text { plagioclase (K-feldspar?) biotite gneiss with vague igneous texture and definite gneissic layering }\end{array}$ \\
\hline OORN131 & 467924 & 7119564 & Fine grained amphibolite \\
\hline OORN143 & 465687 & 7118146 & Hornblende plagioclase gneiss and amphibolite: fine grained, no obvious biotite or garnet \\
\hline OORN148 & 468940 & 7115354 & Napoleon pluton; foliated hornblende monzonite \\
\hline OORN176A & 452555 & 7121884 & Fine grained biotite amphibolite \\
\hline OORN 182 & 455036 & 7121394 & $\begin{array}{l}\text { Chioritized? green coarse-grained biotite homblende quartz monzodiorite orthogneiss, } \text { - interiayered } \\
\text { metasedimentay rocks indicates that the orthogneiss bodies are silks? or very folded }\end{array}$ \\
\hline OORN221 & 459870 & 7121415 & Amphibolite \\
\hline OORN236A & 454129 & 7116222 & Amphibolite with porphyroblastic plagioclase \\
\hline OORN254 & 454002 & 7118386 & Medium-grained, tourmaline muscovite granite \\
\hline OORN271 & 457516 & 7122271 & $\begin{array}{l}\text { Butte Creek pluton: medium- to coarse-grained, porphyritic (big quartz, plagioclase), interstitial K-feldspar biotile } \\
\text { granodiorite }\end{array}$ \\
\hline OORN289B & $4577 \overline{53}$ & 7118659 & Buckskin Creek pluton: medium-grained biolite granodiorile \\
\hline OORN297 & 458469 & 7119197 & Amphibolite and minor amphibole gneiss. \\
\hline OORN330C & 431477 & 7131395 & $\begin{array}{l}\text { Cretaceous? volcanic rock. Friable, greenish biotite feldspar porphyritic andesite? Crystal-lithic tuff with chloritized } \\
\text { biotile }\end{array}$ \\
\hline OORN330D & 431477 & 7131395 & Maroon-weathering hard dacite? crystal-lithic tuff (Cretaceous?) \\
\hline OORN334 & 433313 & 7134650 & Aitered felsic quartz porphyry dike \\
\hline OORN336 & 435419 & $713773 \overline{5}$ & Coarse grained, orange stained quartz porphyry - same dike as 00RN334? \\
\hline OORN337A & $4375 \overline{93}$ & 7140059 & Fine-grained, clay-altered (mostly weathered?) quartz porphyry rhyolite tuff? (Tertiary) \\
\hline OORN337C & 437593 & 7140059 & Vesicular basalt (Tertiary) \\
\hline
\end{tabular}


Table 3. Location and description of rock samples collected in the Eagle and Tanacross quadrangles for major oxide, minor oxide, and trace element analyses.

\begin{tabular}{|l|l|l|l|}
\hline Sample\# & UTWE & UrMN & Sample description \\
\hline OORN420B & 473638 & 7101994 & Fine grained porphyritic basaltic? dike $1 \mathrm{~m}$ wide \\
\hline OORN500A & 475046 & 7100016 & Fine grained biotite amphibolite and amphibolite. Layers from 2 cm to $50 \mathrm{~cm}$ thick \\
\hline O0RN501 & 474862 & 7099943 & Coarse grained quartz diorite? orthogneiss \\
\hline OORN537D & 482820 & 7114162 & Medium grained, quartz feldspar muscovite granite \\
\hline O0RN568A & 486917 & 7108469 & Amphibolite \\
\hline OORN571A & 490525 & 7106945 & Amphibolite and lessor amphibole-plagioclase gneiss \\
\hline OOWM03A & 478585 & 7121530 & Coarse grained orthogneiss \\
\hline OOWM202 & 460648 & 7099958 & Light-colored pluton \\
\hline OOWM321 & 477972 & 7115788 & Amphibolite \\
\hline
\end{tabular}


Tabie 4. Concentration of major-oxides, minor oxides, and trace elements in rock samples from the Eagle and Tanacross quadrangles.

\begin{tabular}{|c|c|c|c|c|c|c|c|c|c|c|c|c|c|c|c|c|c|c|c|}
\hline$\overline{S A M}$ & 203 & $\mathrm{CaO}$ & 203 & $\mathrm{Fe} 203^{*}$ & $\mathrm{~K} 2 \mathrm{O}$ & $\mathrm{MgO}$ & MnO & $\mathrm{Na2O}$ & P205 & $\mathrm{SiO} 2$ & TIO2 & LOI & & $\mathbf{B a}$ & $\mathbf{R b}$ & $\mathrm{Sr}$ & $\mathrm{Nb}$ & $\mathrm{Zr}$ & \\
\hline & $\%$ & $\%$ & $\%$ & $\%$ & $\%$ & $\%$ & $\%$ & $\%$ & $\%$ & $\%$ & $\%$ & $\%$ & $\%$ & $\mathrm{ppm}$ & $\mathrm{ppm}$ & $\mathrm{ppm}$ & $\mathrm{ppm}$ & $\mathrm{ppm}$ & \\
\hline$\overline{\mathrm{DS} 009 \mathrm{~A}}$ & $15.6 \dot{8}$ & 1.62 & $<0.01$ & 0.34 & 0.77 & 0.02 & 0.01 & 6.37 & $<0,01$ & 73.85 & 0.03 & 0.37 & 99.06 & 1170 & 14 & 1015 & 6 & 39 & $\overline{6}$ \\
\hline & 95 & 37 & $<0.0 \overline{1}$ & 91.17 & 1.07 & 7.36 & 1.20 & 3.01 & 0.08 & 9.77 & 1.08 & 0.96 & 9.02 & $7 \overline{85}$ & 22 & 104 & 6 & 72 & \\
\hline & 12 & 46 & 0.01 & 10 & 4.52 & $2 . \overline{25}$ & .11 & 2.51 & .29 & 5.15 & 0.56 & 1.20 & 9.27 & 1675 & 110 & 564 & 12 & 117 & \\
\hline & 16 & 43 & $<0 . \overline{01}$ & .77 & 0.19 & 1.10 & 05 & 0.27 & .08 & 0.24 & 0.18 & 0.38 & 8.85 & 295 & 12 & 36 & 10 & 75 & \\
\hline & 13 & $\overline{04}$ & 01 & 22 & 2.52 & 4.36 & 09 & 1.60 & 17 & 64.74 & 0.94 & 1.05 & 98.86 & 1140 & 56 & 240 & 20 & 141 & 26 \\
\hline 53 & 27 & 86 & $<0.01$ & 2.16 & 4.92 & 0.97 & 0.04 & 3.58 & .04 & 72.66 & 0.19 & 0.39 & 99.08 & 1155 & 122 & 132 & 12 & 96 & 24 \\
\hline $58 \mathrm{~A}$ & 96 & 2.99 & $<0.01$ & 2.38 & 3.32 & 0.67 & 0.06 & 3.83 & 0.13 & 68.81 & 0.25 & 0.63 & 99.03 & 1295 & 90 & 732 & 14 & 132 & 20 \\
\hline $358 \mathrm{~B}$ & 76 & 1.43 & $<0.01$ & 0.63 & 3.85 & $<0.01$ & 0.31 & $\overline{4.41}$ & $\overline{0.02}$ & 73.04 & 0.04 & $0 . \overline{30}$ & 98.79 & 175 & 196 & 140 & 8 & 69 & $6 \overline{4}$ \\
\hline 86 & 34 & 8.83 & $<0.01$ & 10.48 & 1.15 & 6.86 & 0.16 & 2.82 & 0.37 & 48.82 & 1.82 & 2.23 & 98.88 & 435 & 34 & 322 & 18 & 198 & 34 \\
\hline 087 & 39 & 8.80 & $\angle 0.01$ & 10.58 & 1.08 & 6.93 & 0.16 & 2.93 & 0.34 & 48.88 & 1.84 & 2.27 & 99.20 & 435 & 40 & 324 & 16 & 192 & \\
\hline & $2 \overline{8}$ & 86 & $<0.01$ & 5.48 & 2.76 & 2.28 & .11 & 29 & 0.28 & 61.06 & 0.58 & 1.53 & 98.51 & 1305 & 66 & 804 & 16 & 129 & 22 \\
\hline 24 & 62 & $3.1 \overline{2}$ & $\angle 0.01$ & 3.90 & 1.64 & 1.27 & 0.06 & 3.24 & 0.07 & 70.95 & 0.36 & 0.79 & 99.02 & 1105 & 42 & 278 & 10 & 93 & $2 \overline{4}$ \\
\hline 29 & 6.98 & 9.16 & $<0.01$ & 8.90 & 0.74 & 7.18 & 0.16 & 3.87 & 0.28 & 48.75 & 1.16 & 1,38 & $\overline{98.56}$ & $2 \overline{15}$ & 28 & 388 & 8 & $1 \overline{14}$ & 24 \\
\hline $35 \mathrm{~B}$ & 29 & 9.67 & $<0.01$ & 12.27 & 1.94 & 5.03 & 0.24 & 3.39 & 0.82 & 47.77 & 0.88 & 1.77 & 99.07 & 475 & 28 & 892 & 10 & 87 & 84 \\
\hline 41 & $6 \overline{9}$ & 4.59 & $<0.01$ & 5.58 & 5.32 & 1.58 & 0.13 & 4.54 & 0.35 & 7.39 & 0.43 & 0.40 & 9.00 & 2250 & 96 & 1370 & 6 & 108 & 24 \\
\hline $60 \mathrm{~A}$ & 43 & 929 & $<0.01$ & 10.16 & 1.00 & 5.56 & 0.21 & 264 & 0.50 & 47.58 & 217 & 4.22 & 98.76 & $6 \overline{55}$ & 26 & 500 & 22 & 246 & 36 \\
\hline 63 & 30 & 4.12 & $<0.01$ & 3.77 & 2.41 & 1.18 & 0.10 & 16 & 0.19 & 63.43 & 0.46 & 0.76 & 8.88 & 1275 & 50 & 946 & 14 & 126 & 22 \\
\hline DS168 & 3.66 & 1.52 & $<0.01$ & 3.71 & 2.53 & 0.74 & 0.06 & 5.39 & 0.22 & 65.18 & 0.47 & 2.17 & 98.65 & 1600 & 44 & 1150 & 10 & 102 & 14 \\
\hline $\mathrm{DS} 180^{\circ}$ & .36 & 8.46 & $<0.01$ & 11.65 & 1.85 & 4.47 & 0.13 & 2.29 & $0.1 \overline{9}$ & $4 \overline{9} . \overline{2} 6$ & 1.02 & 2.10 & 98.78 & 650 & 52 & 356 & 14 & 78 & 24 \\
\hline 85 & $1 \overline{1}$ & 79 & $<0.01$ & 44 & 2.80 & 08 & & & .16 & $\overline{4.29}$ & 0.35 & 0.70 & & $12 \overline{35}$ & 64 & 34 & 10 & 108 & 18 \\
\hline $15 \mathrm{E}$ & 32 & .84 & $<0.01$ & 7. & 3.20 & 2.98 & 0.09 & & $\overline{0.18}$ & 3.63 & 0.60 & 1.81 & $98 . \overline{73}$ & 675 & 66 & 36 & & 57 & 24 \\
\hline & 18 & & $\angle 0.01$ & 8.92 & 0.61 & 3.3 & 0.09 & & 0.15 & 55.84 & 0.59 & 1.07 & 99.22 & 500 & 20 & 386 & & $4 \overline{8}$ & 18 \\
\hline 3 -6 & 64 & 2.59 & $<0.01$ & 0.85 & 1.39 & 0.06 & 0.01 & 55 & 0.03 & 72.37 & 0.07 & $0 . \overline{28}$ & 98.84 & 1035 & 28 & 1185 & & 75 & 10 \\
\hline $47-6$ & 5.16 & 2.63 & $<0.01$ & 1.29 & 1.77 & 0.21 & 0.02 & 4.80 & 0.06 & 72.51 & 0.14 & 0.38 & 98.97 & 1855 & 36 & 912 & 8 & 99 & 10 \\
\hline $50 \mathrm{~B}-6 / 1$ & 15.19 & 3.88 & $<0.01$ & 3.92 & 3.49 & 1.25 & 11 & 3. & 0.23 & 65.49 & 0.40 & 1.29 & 98.72 & 2430 & 82 & 868 & 16 & 141 & 26 \\
\hline $89-7 / 4$ & 8.75 & 0.72 & $<0.01$ & 0.33 & 3.12 & $<0.01$ & 0.01 & 1.93 & 0.01 & 84.07 & 0.05 & 0.23 & 99.22 & $17 \overline{15}$ & 64 & 312 & 8 & 72 & 12 \\
\hline $94-6 / 20$ & 16 & 86 & $<0.01$ & 19 & 1.68 & 0.19 & 0.02 & 89 & 0.17 & 70.81 & 0.13 & 0.76 & 98.86 & 2020 & 36 & 916 & 8 & 72 & 0 \\
\hline $97-6 / 20$ & 15.07 & 2.49 & $<0.01$ & & 1.84 & 0.32 & 0.03 & & 0.04 & 7250 & 0.10 & 0.64 & 99.08 & 1430 & 44 & 1040 & 6 & 99 & 10 \\
\hline JG125-6/22 & 10.47 & 8.99 & $<0.01$ & 13.58 & 1.50 & 8.01 & 0.17 & 2.38 & 0.53 & 50.70 & 1.39 & 0.99 & 98.71 & 1030 & 36 & 268 & 8 & 54 & 20 \\
\hline JG153-6/25 & 5.32 & 3.85 & $<0.01$ & 2.37 & 1.39 & $0.7 \overline{4}$ & $0.0 \overline{3}$ & & 0.10 & 69.48 & 0.27 & 0.81 & 98.85 & 1405 & 30 & 700 & 8 & 168 & 10 \\
\hline MBW11D & 62 & 3.64 & 01 & 2.84 & 2.08 & 0.82 & 0.04 & 4.28 & 0.20 & 67.61 & 0.30 & 1.20 & 98.63 & 1820 & 48 & 864 & 16 & 123 & 12 \\
\hline DOMBW18 & 3.99 & 4.48 & 0.01 & 5.75 & 3.08 & 2.23 & 0.12 & 3.83 & 0.25 & 59.91 & 0.54 & 1.53 & $\overline{98.71}$ & 1715 & 92 & $\overline{680}$ & 12 & 117 & 22 \\
\hline
\end{tabular}


Table 4. Concentration of major-oxides, minor oxides, and trace elements in rock samples from the Eagle and Tanacross quadrangles.

\begin{tabular}{|c|c|c|c|c|c|c|c|c|c|c|c|c|c|c|c|c|c|c|c|}
\hline SAMF & 1203 & $\mathrm{CaO}$ & 203 & $\mathrm{Fe} 2 \mathrm{O}^{*}$ & $\mathrm{~K} 2 \mathrm{O}$ & $\mathrm{MgO}$ & $\mathrm{MnO}$ & $\mathrm{Na2O}$ & $\mathrm{P} 205$ & S1O2 & TiO2 & LOI & TOTAL & Ba & $\mathbf{R b}$ & $\mathbf{S r}$ & $\mathrm{Nb}$ & $\overline{\mathrm{Z} r}$ & \\
\hline & $\overline{\%}$ & $\%$ & $\%$ & $\%$ & $\%$ & $\%$ & $\%$ & $\%$ & $\%$ & $\%$ & $\%$ & $\%$ & $\%$ & $\overline{\mathrm{ppm}}$ & ppm & ppm & $\mathrm{pPm}$ & ppm & \\
\hline OMBW21 & 13.20 & $0 \overline{88}$ & $<0.01$ & 1.90 & 3.55 & $\overline{0.43}$ & 0.04 & 3.91 & 0.03 & 74.29 & 0.17 & 0.45 & 98.85 & 905 & 100 & 134 & 14 & 105 & 77 \\
\hline V24A & .07 & 66 & 0.01 & 12.39 & 0.67 & 4.12 & .21 & 42 & 0.97 & 49.64 & 2.05 & 0.38 & 98.58 & 160 & 16 & 396 & 10 & $\overline{93}$ & 30 \\
\hline$\sqrt{25 B}$ & 33 & 29 & $\overline{0.01}$ & $3 . \overline{82}$ & 3.21 & 1.37 & 10 & & $0 . \overline{22}$ & 67.73 & 0.42 & 0.76 & & 2080 & $7 \overline{6}$ & 816 & 16 & 135 & \\
\hline$\sqrt{26}$ & .05 & 38 & .01 & & 2.25 & 2.28 & $\overline{13}$ & 56 & $0 . \overline{35}$ & 64.19 & 0.70 & 0.77 & 98.82 & 1825 & 84 & 624 & 16 & 165 & \\
\hline N43 & 39 & $\overline{08}$ & .01 & 4.58 & 3.42 & 1.28 & 0.12 & 3.27 & 0.23 & 65.13 & \begin{tabular}{|l|}
0.46 \\
\end{tabular} & 0.82 & 98.78 & 1560 & $\overline{94}$ & 704 & 16 & 156 & 28 \\
\hline W54 & 9.09 & $7 . \overline{97}$ & 0.01 & $15 . \overline{20}$ & 0.09 & 17.64 & 0.25 & 0.42 & 0.09 & 44.39 & 0.52 & 3.42 & 99.09 & 70 & 14 & 8 & 6 & 33 & 12 \\
\hline$\overline{N 55}$ & 5.48 & $\overline{9.66}$ & $0 . \overline{01}$ & $9 . \overline{49}$ & 0.39 & 8.67 & 0.16 & 3.55 & 0.23 & 47.72 & 1.10 & 1.22 & 98.68 & $12 \overline{5}$ & 18 & 320 & 6 & 102 & 26 \\
\hline & .10 & 7.39 & $<0.01$ & 9.31 & $\{.93$ & $\overline{8.92}$ & $\overline{0.19}$ & 4.05 & $0 . \overline{19}$ & 50.00 & 0.72 & 1.07 & 98.87 & 875 & 46 & 236 & 10 & 75 & 20 \\
\hline W93 & 51 & 02 & 0.01 & $1 . \overline{61}$ & 2.37 & 0.75 & 0.06 & $\overline{5.82}$ & $0 . \overline{15}$ & $66.8 \overline{2}$ & 0.14 & .88 & & 1935 & 38 & $\{060$ & 6 & 78 & 20 \\
\hline N97 & .94 & $\overline{79}$ & $\overline{01}$ & $3.2 \overline{6}$ & 3.81 & 1.04 & 0.08 & 3.51 & 0.19 & 67.76 & 035 & 1.32 & 99.05 & 4580 & 96 & 694 & 12 & 126 & 22 \\
\hline$\sqrt{ } 12 \bar{T}$ & .01 & 05 & 01 & $5 . \overline{98}$ & 4.63 & 1.68 & 0.14 & 4.32 & $0 . \overline{35}$ & 57.06 & 0.46 & 0.41 & 99.09 & 2560 & 86 & 1355 & 8 & 93 & 22 \\
\hline V126 & 28 & 56 & 01 & 7.12 & 4.37 & 2.35 & 0.16 & 4.48 & 0.45 & 54.98 & 0.64 & $\overline{0} .51$ & 98.90 & 2210 & 74 & 1295 & 12 & 135 & 24 \\
\hline$N 141$ & 5.15 & 2.18 & $<0.01$ & 0.79 & 3.69 & 0.26 & 0.04 & 3.73 & 0.03 & 72.32 & 0.09 & $\overline{0.57}$ & $98 . \overline{85}$ & $300 \overline{0}$ & 64 & 1030 & 10 & 60 & 12 \\
\hline W143 & .22 & 1.29 & $<0.01$ & 0.35 & 1.50 & 0.07 & 0.01 & $\overline{7.23}$ & $0 . \overline{01}$ & $70 . \overline{1}$ & 0.04 & 0.51 & 98.94 & 1305 & 40 & 988 & 6 & 30 & 8 \\
\hline$W 215 \mathrm{~A}$ & 62 & 4.80 & $<0.01$ & $6 . \overline{97}$ & 3.40 & 2.99 & 0.13 & 2.33 & 0.41 & 62,01 & 85 & 92 & $98 . \overline{4} 3$ & 2550 & 84 & 590 & 16 & 180 & 28 \\
\hline 216 & 41 & 54 & $<0.01$ & 6.39 & 2.50 & 1.89 & 0.16 & 3.96 & 0.28 & 58.77 & 0.55 & 0.67 & 99.12 & 1190 & 54 & 874 & 12 & 144 & 24 \\
\hline 243 & .05 & $8 . \overline{28}$ & $<0 . \overline{01}$ & $11 . \overline{09}$ & 0.99 & 6.65 & 0.36 & 2.94 & 0.34 & $48.2 \overline{1}$ & 1.88 & 3.34 & 98.93 & 490 & 30 & 290 & 16 & 255 & 36 \\
\hline$\sqrt{313}$ & .86 & 7.62 & $<0.01$ & 11.04 & 1.69 & 4.73 & 0.13 & $\overline{3.15}$ & $0 . \overline{51}$ & 50.68 & 2.42 & 2.21 & 99.04 & 660 & 50 & 294 & 24 & 282 & $4 \overline{4}$ \\
\hline$\sqrt{318}$ & 66 & 1.65 & 0.01 & 3.71 & 5.03 & 0.17 & 0.05 & 2.83 & 0.10 & 70.31 & 0.43 & 1.99 & 98.93 & 1790 & 198 & 128 & 36 & 498 & 76 \\
\hline N445B & .79 & 8.11 & $<0.01$ & 10.47 & 0.31 & 5.78 & $\overline{0.16}$ & 2.83 & $0 . \overline{32}$ & 45.76 & 1.79 & 8.14 & 98.46 & 335 & $\overline{12}$ & 392 & 20 & 264 & 36 \\
\hline$\sqrt{474}$ & 99 & $2 . \overline{63}$ & $<0.01$ & $1.7 \overline{7}$ & 2.00 & 0.44 & 0.04 & 4.78 & 0.06 & 70.61 & 0.18 & 0.47 & 98.97 & 1735 & 50 & 752 & 10 & 108 & 10 \\
\hline N507 & .57 & 0.63 & $<0 . \overline{01}$ & $1.4 \overline{1}$ & 4.50 & 0.17 & 0.03 & 2.96 & 0.03 & 76.03 & 0.11 & $\overline{0} .53$ & 98.97 & 1440 & 146 & 78 & 14 & 120 & 40 \\
\hline 1535 & 80 & 3.01 & $<0 . \overline{01}$ & 4.62 & 4.58 & 1.55 & 0.09 & 2.49 & 0.28 & 65.50 & 0.49 & 1.48 & 98.89 & 1970 & 86 & 684 & 14 & 159 & 26 \\
\hline N570 & 14 & 8.13 & $<0.01$ & 9.57 & 1.35 & 5.86 & 0.11 & $\overline{3.22}$ & $0 . \overline{15}$ & 49.67 & 0.64 & 2.18 & 99.02 & 700 & 36 & 342 & 8 & 57 & 14 \\
\hline N575 & .25 & 8.47 & $<0.01$ & 8.20 & 1.10 & 4.54 & 0.12 & 4.31 & $0 . \overline{34}$ & 52.78 & 1.15 & 0.70 & 98.96 & 360 & 32 & 276 & 12 & 144 & 30 \\
\hline & .85 & 44 & 0.01 & $2 . \overline{99}$ & 4.32 & 1.07 & 0.07 & 2.59 & 0.15 & 70.32 & 0.65 & 1.43 & 98.88 & 855 & 178 & 148 & 22 & 225 & 44 \\
\hline & 72 & 5.49 & 0.01 & $8.5 \overline{8}$ & 0.40 & 1.96 & 0.07 & 4.77 & 0.35 & 60.84 & 0.96 & 0.40 & $98 . \overline{54}$ & $63 \overline{5}$ & 14 & 406 & 4 & 63 & 24 \\
\hline & 15.18 & 2.65 & $\angle 0.01$ & 0.64 & 2.17 & 0.15 & 0.01 & $\overline{4.49}$ & $0 . \overline{05}$ & 72.94 & 0.08 & 0.42 & 98.78 & 3140 & 40 & 892 & 6 & 75 & 6 \\
\hline $00 \mathrm{RI}$ & 8.74 & 0.47 & $<0.01$ & 1.65 & 4.01 & 0.48 & 0.03 & 1.29 & 0.05 & 81.43 & 0.20 & 0.71 & 99.06 & 925 & 104 & 108 & 8 & 144 & 16 \\
\hline$\overline{O O R N}$ & .87 & $6 . \overline{44}$ & $<0.01$ & 13.81 & 0.40 & 3.77 & 0.21 & 4.17 & 0.06 & 54.90 & 0.76 & 0.23 & 98.62 & 165 & 12 & 140 & 4 & 39 & 18 \\
\hline OORA & 44 & .97 & $<0.01$ & 11.19 & 0.41 & 6.81 & 0.19 & 3.49 & 0.19 & 50.44 & 1.00 & 0.73 & 98.86 & $15 \overline{5}$ & 12 & 302 & 6 & 63 & 20 \\
\hline 00RN73 & 17.19 & 5.79 & $<0.01$ & 5.16 & 2.38 & 1.96 & 0.11 & 3.57 & 0.24 & 61.54 & \begin{tabular}{|l|}
0.47 \\
\end{tabular} & $\overline{0} .65$ & 99,06 & 1320 & 60 & 990 & 12 & 126 & 18 \\
\hline
\end{tabular}


Table 4. Concentration of major-oxides, minor oxides, and trace elements in rock samples from the Eagle and Tanacross quadrangles.

\begin{tabular}{|c|c|c|c|c|c|c|c|c|c|c|c|c|c|c|c|c|c|c|c|}
\hline SAMPLE & $\mathrm{Al} 2 \mathrm{O} 3$ & $\mathrm{CaO}$ & $\mathrm{Cr} 2 \mathrm{O} 3$ & $\mathrm{Fe} \mathrm{OO3}^{*}$ & K20 & $\mathrm{MgO}$ & MnO & $\mathrm{Na2O}$ & P205 & SiO2 & $\overline{\mathrm{TIO} 2}$ & LOI & TÖTAL & $\mathrm{Ba}$ & $\mathbf{R b}$ & $\mathrm{Sr}$ & $\mathrm{Nb}$ & $\mathrm{Zr}$ & $Y$ \\
\hline & $\%$ & $\%$ & $\%$ & $\%$ & $\%$ & $\%$ & $\%$ & $\%$ & $\%$ & $\%$ & $\%$ & $\%$ & $\%$ & $\mathrm{ppm}$ & $\mathrm{ppm}$ & $\mathrm{ppm}$ & ppmi & $\mathrm{ppm}$ & ppm \\
\hline $00 R$ N75 & 14.67 & 4.58 & $<0.01$ & 6.22 & 4.27 & 2.27 & 0.12 & 2.02 & 0.36 & 62.41 & 0.69 & 0.94 & 98.55 & 2540 & 98 & 676 & 14 & 177 & 30 \\
\hline OORN84 & 14.27 & $\overline{4.47}$ & $<0.01$ & 5.93 & 4.24 & 2.21 & 0.11 & 1.93 & 0.45 & 63.29 & 0.72 & 1.03 & 98.65 & 2220 & 100 & 594 & 16 & 162 & 30 \\
\hline OORN93 & 14.62 & 4.52 & $<0 . \overline{01}$ & 5.59 & 3.71 & 1.95 & $\overline{0.11}$ & 2.21 & 0.33 & 64.18 & 0.62 & 1.03 & 98.87 & 2090 & 88 & 668 & 36 & 177 & 30 \\
\hline 00RN96A & 18.90 & $\overline{6.42}$ & $<0 . \overline{01}$ & 9.20 & 1.50 & 3.30 & $\overline{0.15}$ & 4.59 & $0 . \overline{14}$ & 53.30 & 0.82 & 0.76 & 99.08 & 935 & 32 & 348 & 8 & 81 & 24 \\
\hline DORN96B & 16.01 & 3.02 & $<0.01$ & 2.67 & 2.20 & 0.75 & 0.04 & 4.08 & $0 . \overline{19}$ & 68.45 & 0.28 & 0.78 & 98.47 & 1945 & 48 & 782 & 20 & 126 & 14 \\
\hline 00RN99 & 13.59 & 3.95 & $<0 . \overline{01}$ & 4.69 & 2.13 & 1.25 & 0.08 & 2.39 & 0.08 & 69.31 & 0.41 & 0.73 & 98.61 & 1065 & 62 & 190 & 14 & 120 & 26 \\
\hline oORN131 & 45.31 & 6.25 & $<0.01$ & 9.90 & 0.97 & 3.58 & 0.18 & 5.09 & 0.69 & $\overline{54.43}$ & 1.59 & 1.27 & 99.26 & 365 & 26 & 246 & 14 & 120 & 32 \\
\hline OORN143 & 18.62 & 12.02 & $<0.01$ & 10.52 & 0.88 & $4 \overline{50}$ & 0.17 & 3.24 & 0.16 & 46.65 & 0.86 & 1.38 & 99.00 & $210^{-7}$ & 18 & 270 & 2 & 48 & 16 \\
\hline OORN 148 & $\overline{18.12}$ & 4.89 & $<0.01$ & 6.10 & 4.66 & 1.75 & 0.15 & 4.29 & 0.38 & 57.75 & 0.48 & 0.52 & 99.09 & 2310 & 82 & 1345 & 8 & 78 & 24 \\
\hline OORN176A & 15.22 & 7.41 & $<0.01$ & 9.13 & 2.87 & 5.20 & 0.17 & $\overline{2.61}$ & $0 . \overline{36}$ & 53.87 & 0.88 & 1.12 & 98.84 & 3150 & $60^{-}$ & 480 & 10 & 117 & 26 \\
\hline OORN 182 & 14.24 & 5.94 & $<0.01$ & $\overline{7,10}$ & 3.11 & 3.16 & 0.15 & 2.91 & $0 . \overline{39}$ & 59.95 & 0.77 & 0.94 & 98.66 & 2100 & 48 & 1020 & 16 & 177 & 30 \\
\hline O0RN221 & 16.67 & 5.35 & $<0.01$ & 9.03 & 1.01 & 407 & 0.22 & 3.75 & 0.20 & 56.16 & 0.81 & 1.58 & 98.85 & 280 & 28 & 312 & 6 & 81 & 30 \\
\hline O0RN236A & 16.67 & 6.57 & $<0.01$ & 8.26 & 3.01 & 4.31 & 0.16 & 4.20 & 0.17 & 53.45 & 0.64 & 7.59 & 99.03 & 1730 & 48 & 564 & 8 & 63 & 14 \\
\hline OORN254 & 15.51 & $\overline{1.86}$ & $<0.01$ & 0.62 & 2.41 & 0.19 & 0.03 & 3.52 & 0.09 & 73.16 & 0.09 & 0.85 & 98.33 & 1065 & 66 & 250 & 10 & 42 & 42 \\
\hline OORN271 & 14.86 & 3.59 & $<0.01$ & 3.76 & 3.28 & 1.19 & 0.09 & 2.72 & 0.22 & 67.24 & 0.39 & 1.19 & 98.53 & 1695 & 70 & 722 & 14 & 153 & 24 \\
\hline OORN289B & 14.99 & $\overline{2.72}$ & $<0.01$ & 2.91 & 3.35 & 0.97 & 0.08 & 3.42 & 0.15 & 68.41 & 0.33 & 1.33 & 98.66 & 2310 & 82 & 768 & 14 & 117 & 20 \\
\hline 00RN297 & 15.36 & 5.17 & $<0.01$ & 7.83 & 1.80 & 4.24 & $\overline{0.14}$ & 3.11 & 0.12 & 58.53 & 0.64 & 1.89 & 98.83 & $7 \overline{775}$ & 42 & 302 & 8 & 96 & 24 \\
\hline $00 \mathrm{RN} 330 \mathrm{C}$ & 14.02 & 2.49 & $<0.01$ & 2.44 & 2.53 & 102 & 0.06 & 2.07 & 0.11 & 68.89 & 0.34 & 4.64 & 98.61 & 835 & 110 & 394 & 20 & 159 & 20 \\
\hline OORN330D & $\$ 4.85$ & 1.78 & $<0.01$ & 2.75 & 4.11 & 0.84 & 0.04 & 2.73 & 0.11 & 69.38 & 0.35 & 2.17 & 99.11 & $\{\overline{825}$ & 134 & $\overline{436}$ & 20 & 162 & 22 \\
\hline $008 N 334$ & 12.67 & 0.22 & $\angle 0 . \overline{01}$ & 1,13 & 5.15 & 0.18 & 0.01 & 2.89 & 0.01 & 75.45 & 0.08 & 1.17 & 98.96 & 160 & 450 & 6 & 70 & 201 & 120 \\
\hline$\sqrt{336}$ & 12.26 & 0.05 & $<0.01$ & 1.22 & 5.40 & $<0.01$ & $<0.01$ & 2.62 & 0.04 & 75.97 & 0.26 & \begin{tabular}{|l|}
1.17 \\
\end{tabular} & 98.99 & 885 & $25 \overline{0}$ & 28 & 40 & 366 & 48 \\
\hline 00RN337A & 11.80 & 0.89 & $<0.01$ & 0.94 & 5.31 & $<0 . \overline{1}$ & $<0.01$ & 2.21 & 0.09 & 76.29 & 0.41 & 1.03 & 98.97 & 1395 & 224 & 96 & 34 & 438 & 76 \\
\hline $00 \mathrm{RN} 337 \mathrm{C}$ & 14.32 & 9.57 & $<0 . \overline{01}$ & 11.69 & 1.24 & 4.35 & 0.18 & 2.98 & 0.48 & 47.08 & 2.31 & 4.65 & 98.85 & 560 & 26 & 286 & 22 & 240 & 38 \\
\hline $\mathrm{N} 420 \mathrm{~B}$ & 14.40 & 4.27 & 0.07 & 7.78 & 6.74 & 9.73 & 0.12 & 3.70 & 0.90 & 49.21 & 0.77 & 0.91 & 98.60 & 3560 & 186 & 1150 & 14 & 153 & 26 \\
\hline $0 \overline{0 R N 500 A}$ & 16.53 & 6.10 & $<0 . \overline{01}$ & 8.57 & 1.20 & 5.88 & 0.15 & 5.41 & 0.19 & 52.66 & 0.88 & 1.26 & 98.83 & 400 & 26 & 218 & 6 & 87 & 24 \\
\hline 00RN501 & 16.56 & 3.24 & $<0.01$ & 3.06 & 2.59 & 1.03 & 0.04 & 4.44 & 0.11 & 66.33 & 0.40 & 0.86 & 98.66 & 3150 & 60 & 618 & 14 & 108 & 12 \\
\hline O0RN537D & 15.39 & 0.93 & $<0.01$ & 0.23 & 4.05 & $<0 . \overline{01}$ & 0.01 & 5.47 & $<0.01$ & 72.07 & 0.04 & 0.17 & 98.36 & 1035 & 78 & 406 & 6 & 30 & 10 \\
\hline$\sqrt{568 \mathrm{~A}}$ & 14.14 & 6.89 & $<0.01$ & 13.24 & 0.90 & 6.83 & 0.23 & 4.28 & 0.20 & 49.29 & 1.93 & 0.85 & 98.78 & 290 & $2 \tilde{2}$ & 148 & 6 & 84 & 30 \\
\hline 00RN571A & $11 . \overline{82}$ & 5.78 & $<0.01$ & 8.85 & 1.84 & 4.81 & 0.17 & 2.68 & 0.11 & 61.12 & 0.79 & 0.91 & 98.88 & 1255 & 44 & 176 & 6 & 66 & 24 \\
\hline OOWMO3A & 15.99 & 3.31 & $<0.01$ & $\overline{1.43}$ & 1.87 & $0 . \overline{40}$ & 0.03 & 4.72 & 0.06 & 69.88 & 0.13 & 0.59 & 98.41 & 1165 & 44 & 800 & 12 & 90 & 10 \\
\hline 00WM202 & 17.16 & 3.43 & $<0.01$ & 2.54 & 2.22 & 0.77 & 0.07 & 5.63 & 0.14 & 66.05 & 0.30 & 0.47 & 98.78 & 1450 & 46 & 906 & 16 & 93 & 14 \\
\hline 00 WM321 & 12.06 & $\overline{10.02}$ & 0.01 & 19.26 & 2.38 & 9.57 & 0.26 & 1.39 & 0.24 & 40.33 & 1.91 & 1.33 & 98.76 & 1300 & 74 & 218 & 10 & 39 & 22 \\
\hline
\end{tabular}


Table 5. Concentration of rare earth elements in rock samples from the Eagle quadrangle.

\begin{tabular}{|c|c|c|c|c|c|c|c|c|c|c|c|c|c|c|c|c|c|}
\hline SAMPLE & $\begin{array}{l}\mathrm{Ce} \\
\mathrm{ppm}\end{array}$ & $\begin{array}{l}\text { Dy } \\
\text { ppm }\end{array}$ & $\begin{array}{l}\text { Er } \\
\text { gppm }\end{array}$ & $\begin{array}{l}\text { Eu } \\
\text { pppm }\end{array}$ & $\begin{array}{c}\text { Gd } \\
\mathrm{ppm}\end{array}$ & $\begin{array}{l}\text { Ho } \\
\text { ppm }\end{array}$ & $\begin{array}{l}\text { La } \\
\mathrm{ppm}\end{array}$ & $\begin{array}{l}\text { Lu } \\
\mathrm{ppm}\end{array}$ & $\begin{array}{l}\mathrm{Nd} \\
\mathrm{ppm}\end{array}$ & $\begin{array}{c}\mathrm{Pr} \\
\mathrm{ppm}\end{array}$ & $\begin{array}{c}\mathrm{Sm} \\
\mathrm{ppm}\end{array}$ & $\begin{array}{c}\text { Th } \\
\text { ppmm }\end{array}$ & $\begin{array}{c}\text { Th } \\
\text { ppm }\end{array}$ & $\begin{array}{l}T \mathrm{Tm} \\
\mathrm{ppm}\end{array}$ & $\begin{array}{c}\mathrm{U} \\
\mathrm{ppm}\end{array}$ & $\begin{array}{c}\mathrm{Y} \\
\mathrm{ppm}\end{array}$ & $\begin{array}{l}\text { Yb } \\
\text { ppm }\end{array}$ \\
\hline $99 M B W 256$ & 5.0 & 1.0 & 0.7 & $0 . \overline{3}$ & 1.0 & 0.2 & 2.5 & 0.1 & 3.0 & 0.6 & 0.8 & 0.1 & $<1$ & 0.1 & $<0.5$ & 7.5 & 0.7 \\
\hline$\overline{99 R A}$ & 0.0 & 6 & 1.6 & $\overline{0.7}$ & 2.7 & 0.5 & 11.0 & $\overline{0.2}$ & 9.5 & 2.5 & 2.1 & 0.4 & 1 & 0.2 & 0.5 & 17.0 & $\overline{1} . \overline{7}$ \\
\hline 99RI & 5 & 3 & 2.5 & 1.3 & 5.0 & 0.9 & 7.5 & 0.3 & 15.0 & 3.3 & 4.0 & 0.7 & $<1$ & $\overline{0} . \overline{4}$ & 1 & 27.5 & 2.5 \\
\hline 99RN & 4.0 & .0 & $2 . \overline{0}$ & 0.7 & 2.8 & 0.7 & 6.0 & $\overline{0.3}$ & 8.0 & 1.8 & 2.1 & 0.5 & $<1$ & 0.3 & $<0.5$ & 21.0 & 2.0 \\
\hline 99RN & 1.5 & 1 & 3.2 & 1.2 & 4.4 & 1.1 & 9.0 & $\overline{0.5}$ & 11.5 & 2.7 & 3.4 & 0.8 & 3 & 0.4 & 1 & 33.5 & 3.4 \\
\hline DS & .5 & 4 & & 1.2 & 3.7 & 0.7 & 11.5 & $\overline{0 . j}$ & 14.0 & 3.6 & 3.0 & 0.6 & $<1$ & 0.3 & $<0.5$ & 20.5 & 1.8 \\
\hline$\overline{D S}$ & 5 & 2.8 & 1.7 & 0.8 & $\overline{2.9}$ & 0.6 & 7.5 & 0.2 & 8.5 & 2.0 & 2.1 & 0.4 & $<1$ & $\overline{0} . \overline{2}$ & 0.5 & 18.5 & 1.7 \\
\hline $5-6 / 22$ & 18.5 & & 2.6 & & 4.3 & $\overline{0} . \overline{8}$ & 8.5 & 0.2 & 35 & 2.9 & 3.6 & $\widehat{0.6}$ & 2 & $\overline{0.3}$ & $\overline{1}$ & 18.5 & 1.7 \\
\hline 55 & 19.0 & & .8 & & 3.4 & 0.7 & 7.5 & 0.3 & 11.5 & 2.7 & 2.8 & 0.5 & $<1$ & 0.2 & $<0.5$ & 19.5 & 1.8 \\
\hline & $\overline{5.5}$ & & 16 & 5 & 1. & 0.5 & 2.0 & 0.2 & & $0 . \overline{8}$ & 1.4 & 1.3 & $<1$ & 0.2 & $<0.5$ & 16.0 & 1.7 \\
\hline & 1.5 & & 31 & 0 & 3.5 & 0.7 & 6.5 & 0.3 & 11.0 & 2.1 & 2.7 & 0.6 & $<1$ & 0.3 & $<0.5$ & 19.5 & 2.0 \\
\hline & & & & 19 & 3.2 & 0.6 & 7.5 & $\overline{0.3}$ & 10.5 & 2.6 & 2.8 & 0.5 & $<1$ & 0.3 & 0.5 & 16.5 & 1.8 \\
\hline & & & & & 3. & 0.7 & 7.0 & 0.3 & 90 & 2.1 & $\overline{2.3}$ & 0.5 & $\overline{1}$ & 0.3 & 0.5 & 78.5 & 2.0 \\
\hline OORN586A & 17.0 & 4.8 & $\overline{3.2}$ & 1.3 & 4.6 & 9.0 & $6 . \overline{5}$ & 0.4 & 11.5 & 2.6 & 3.4 & 0.8 & $<1$ & 0.4 & 0.5 & 31.0 & 3.4 \\
\hline
\end{tabular}

